\title{
How bees distinguish patterns by green and blue modulation
}

This article was published in the following Dove Press journal:

Eye and Brain

5 October 2015

Number of times this article has been viewed

\section{Adrian Horridge}

Biological Sciences, Australian National University, Canberra, $\mathrm{ACT}$, Australia
Correspondence: Adrian Horridge 76 Mueller Street, Yarralumla, Canberra, ACT 2600, Australia Email horridge@netspeed.com.au
Abstract: In the 1920s, Mathilde Hertz found that trained bees discriminated between shapes or patterns of similar size by something related to total length of contrasting contours. This input is now interpreted as modulation in green and blue receptor channels as flying bees scan in the horizontal plane. Modulation is defined as total contrast irrespective of sign multiplied by length of edge displaying that contrast, projected to vertical, therefore, combining structure and contrast in a single input. Contrast is outside the eye; modulation is a phasic response in receptor pathways inside. In recent experiments, bees trained to distinguish color detected, located, and measured three independent inputs and the angles between them. They are the tonic response of the blue receptor pathway and modulation of small-field green or (less preferred) blue receptor pathways. Green and blue channels interacted intimately at a peripheral level. This study explores in more detail how various patterns are discriminated by these cues. The direction of contrast at a boundary was not detected. Instead, bees located and measured total modulation generated by horizontal scanning of contrasts, irrespective of pattern. They also located the positions of isolated vertical edges relative to other landmarks and distinguished the angular widths between vertical edges by green or blue modulation alone. The preferred inputs were the strongest green modulation signal and angular width between outside edges, irrespective of color. In the absence of green modulation, the remaining cue was a measure and location of blue modulation at edges. In the presence of green modulation, blue modulation was inhibited. Black/white patterns were distinguished by the same inputs in blue and green receptor channels. Left-right polarity and mirror images could be discriminated by retinotopic green modulation alone. Colors in areas bounded by strong green contrast were distinguished as more or less blue than the background. The blue content could also be summed over the whole target. There were no achromatic patterns for bees and no evidence that they detected black, white, or gray levels apart from the differences in blue content or modulation at edges. Most of these cues would be sensitive to background color but some were influenced by changes in illumination. The bees usually learned only to avoid the unrewarded target. Exactly the same preferences of the same inputs were used in the detection of single targets as in discrimination between two targets.

Keywords: color vision, honey bee, sensory processing, place recognition, detector design

\section{Introduction}

Studies of bees (Apis mellifera) that distinguish colored targets or flowers in search of a reward of sugar have been published for at least 100 years, ${ }^{1}$ but the emphasis has been on descriptions of the abilities, or performance, rather than the identification of preferred inputs that the bees detected. The honeybee has three types of receptor cells behind each facet of the compound eye, with peaks in the ultraviolet (UV), blue, and green parts of the spectrum. ${ }^{2}$ The intensity of the stimulus at each of the three types 
may be calculated or measured experimentally, but it tells us nothing about the subsequent processing.

Electrophysiology of insect optic lobes has provided a bewildering complexity of high-speed neuron responses, but told us little about mechanisms of vision or what bees actually detected. The justification for this statement is that electrophysiology was never able to prove or disprove the trichromatic theory of color vision in any insect. However, conclusions from training and testing must be compatible with physiological findings. Recording from the honeybee revealed that steady illumination adapted receptor cells to a high plateau within 1 second and amplified the modulation relative to steady tonic parts of the signal. ${ }^{3}$ At the next level in the insect lamina, the largest and most abundant secondorder neurons adapt rapidly to a low plateau and further amplify the modulation. The overall effect is to optimize the detection of modulation (not contrast) when scanning across edges. ${ }^{4}$ Calculation suggests that responses of lamina neurons are tuned to detect edges and small spots, not areas of black, white, or color. ${ }^{5}$ As a bee scans in the horizontal plane in flight, visual receptors and peripheral neurons are necessarily modulated by contrasts, contours, and edges of patterns. The term figural intensity was the name given long ago, when it was discovered that two shapes of similar size were easily discriminated the more they differed in the total length of edge. ${ }^{6}$ Figural intensity is now interpreted as the summed modulation of the receptor responses by contrasts at vertical edges as flying bees scan in the horizontal plane. Until better calibrated, it is measured as total length of edge projected to the vertical, multiplied by the contrast at each piece of edge. $^{7}$

Neuron anatomy of the insect visual system has revealed many neuron connexions, but little about the mechanisms of vision. The justification for this statement is that neuron anatomy was never able to prove or disprove the trichromatic theory of color vision. However, conclusions derived from testing trained bees must be compatible with all anatomical findings. All six axons of the green receptors in each ommatidium have been reported as ending at the lamina level. ${ }^{8}$ Therefore, it is probable that the green channel carries no persistent tonic signal, in which case color vision could not be trichromatic. Motion detection is done entirely by the green receptors..$^{9}$ Axons of receptors that bypass the lamina ${ }^{8}$ with spectral peaks in the blue and UV could, however, carry signals from steady illumination of homogeneous areas. In the training and testing experiments below, UV was excluded.

In recent works, bees trained on gratings revealed the limits of spatial resolution. ${ }^{10}$ The lower limit for horizontal versus vertical gratings in either green or blue pathways was $2.5^{\circ}$, but resolution of green contrast was reduced by blue contrast. ${ }^{11}$ Later, it was found that the orientation detectors occurred only in the green receptor channel, ${ }^{12}$ so in gratings equiluminant for green receptors, bees measured the resolution of blue modulation detectors, not orientation. Subsequently, it was realized that when presented with horizontal versus vertical patterns, bees preferred to learn a measure of green modulation rather than orientation ${ }^{13}$ and the extreme resolution of single green receptors was approximately $2^{\circ}$. Orientation detectors (three ommatidia wide) had a slightly larger resolution and required an edge length of approximately $3^{\circ}$, and therefore were three facets long on the retina. ${ }^{14}$ Modulation detectors were symmetrical, as shown by the failure to notice when a black/white boundary was replaced by a white/black one at the same place. ${ }^{15-17}$

More recent works show that bees trained to distinguish between colors detected and located and measured only two or three simple features. ${ }^{16,17}$ One was the content of blue, which is the tonic response of the blue receptor pathway measured over a large field, probably over each eye separately. The other was the modulation of green receptor pathways that enabled bees to locate edges, measure modulation, and measure angular width between vertical edges. ${ }^{16}$ In the absence of green contrast, green modulation was replaced by the less-preferred blue modulation. ${ }^{16,17}$ Relative positions of blue content and receptor modulation enabled the detection of left-right polarity, like a signpost. ${ }^{17}$ There was no suggestion of trichromatic color vision and much evidence against. $^{16-18}$

The effort so far has been to reveal signals that passed along green- and blue-sensitive pathways from the simplest displays. Next, coincidences of two known inputs from edges and areas will be examined in some interesting patterns, notably the measure of angular subtense of bars and whole patterns, location of modulation, suppression of blue modulation by green modulation, and the detection of asymmetry. Remaining questions are whether all feature detectors have been found and whether more complex patterns will require new coincidences. On the other hand, we may have found the limits of the peripheral visual inputs.

In several previous efforts, ${ }^{16-18}$ a tonic green receptor input was sought but not revealed. Therefore, in the absence of UV, bees have three independent variables available in the signal at the receptor level. They are the tonic (persistent) responses in the blue channel and the phasic (rapidly decaying modulation) responses of the blue and the green receptor channels. ${ }^{16-18}$ 
The variables were further reduced in number by working with patterns that were equiluminant for the green or alternately for the blue receptors, so that inputs to blue and green channels were isolated. The target patterns were further designed to display quantitative differences in blue content or in green or blue modulation separately.

\section{Materials and methods}

The apparatus and methods have been described many times. ${ }^{11-18}$ The apparatus (Figure 1) was made of polycarbonate sheet with wooden sides and a transparent top, which excluded UV. A transparent plastic roof above provided further protection. The floor and inside walls of the apparatus were painted flat white with poor UV reflection. The UV was further reduced by the use of common white or colored papers that reflected negligible UV.

At a range of $27 \mathrm{~cm}$, the bees had a choice between two targets subtending up to $55^{\circ}$. After making their choice, bees were obliged to fly over one of the baffles of thin polycarbonate film and later made their exit by the same route.

The reward was a solution of cane sugar that was adjusted in strength between $2 \%(\mathrm{w} / \mathrm{w})$ and $7 \%(\mathrm{w} / \mathrm{w})$ so that recruits were not attracted, but marked bees continued to return for more. The pattern, with the reward, changed sides every 5 minutes to prevent teaching which side to go and to equalize any chance of spurious cues from unequal olfactory cues or side preferences. Spurious results were avoided by testing with two clean identical targets or with a reward on both sides. A reward was provided in the test phase; otherwise, bees continued to search. Test phases were for 5 minutes, separated by continued training for 20 minutes. At a different time, the test was repeated with the two sides reversed. Each test was followed by more training and then by a different test. The many different tests implied a long gap before any one test was repeated; hence, the bees learned only the training display. Training interrupted by tests continued all week until sufficient counts accumulated.

Gray papers were printed with a high-quality laser printer. To be consistent with the calibrations of colored papers, gray papers were identified by the percentage of white, not black. Canson-colored papers were purchased from an art supply shop and can be ordered online at http://www.canson-infinity. com/en/values.asp. To avoid confusion about bee colors and names in the literature, the color names of this paper

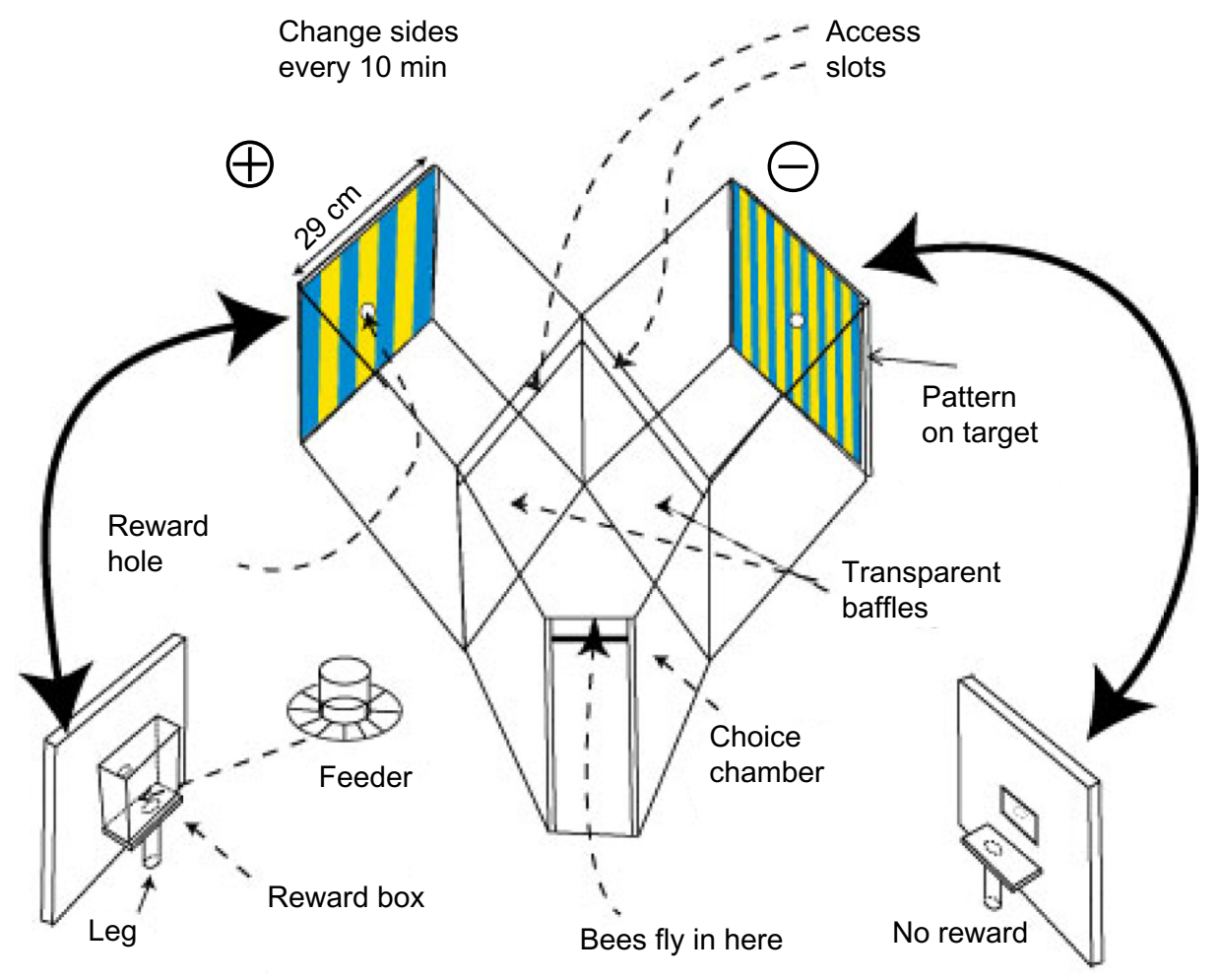

Figure I The Y-choice apparatus provides complete control of the stimulus.

Notes: The bees entered through a narrow horizontal slot at the top front into a choice chamber from which they could see both targets. They selected one side, passed over one of the transparent baffles via the narrow horizontal slot, and reached the reward hole. When satisfied, they exited by the way they came. To make the bees look at the patterns and learn something, the reward with its pattern changed sides every 5 min or 10 min. Insets show behind the targets, with the reward box and the feeder located behind the rewarded target.

Abbreviation: min, minutes. 
manufacturer have been used. Details of the stimulus to the blue and green receptors reflected from these papers are given in Table 1 and in several previous works. ${ }^{19,20}$ Methods of calibration are given in the literature. ${ }^{11-13}$

\section{Statistics}

Scores at each test are presented as the percentage of correct choices, followed by the number of choices by a group of 12-15 trained bees. The test scores were not comparable to each other because each is a forced choice between two unfamiliar targets with different cues in each; so in an ideal world, the bees would be $50 \%$ (fail) or $100 \%$ correct. Therefore, it is hard to justify that differences between intermediate scores have any validity. Conclusions were deduced by logic, based on whether the bees could or could not pass the tests in each experiment. A variety of different training experiments supported each other, with numerous tests in each experiment.

With continued training and other tests intervening, each test was continued until 100-200 counts had been made. Because the data are frequencies, standard deviations were calculated from the following formula: $\mathrm{sd}=\sqrt{ }[P(1-P) / \mathrm{n}]$, where $P$ is the measure of probability of a correct response and $n$ is the number of observations. ${ }^{15}$ This formula is valid when the choices of the bees are independent and the scores have no trend. In each test, only a significant pass or fail was required, so we need to know whether each test score was different from $50 \%$. As a quick rule of thumb, a score of more than $0.57(57 \%)$ for $n=200$ or a score of $0.60(60 \%)$ for $n=100$ was more than two standard deviations greater than chance $(P<0.05)$, which was acceptable.

In the more exact method, we wish to reject the null hypothesis that the observed score is not different from $50 \%$ in a

Table I Relative receptor excitations by different papers relative to white paper (100\%) and contrasts between two pairs of papers used in equiluminance experiments

\begin{tabular}{lll}
\hline Canson color name & Blue receptor & Green receptor \\
\hline White copy paper & 100 & 100 \\
Hemp 374 & 34.2 & 56.3 \\
Ultramarine 590 & 33.8 & 20.7 \\
Billiards green 576 & 17.0 & 22.3 \\
Buff 384 & 25.7 & 41.7 \\
Blue 595 & 54.2 & 40.0 \\
Dresden yellow & 13.1 & 78.1 \\
Contrast 374/590 & $\mathbf{0 . 0 6}$ & 0.46 \\
Contrast 384/595 & 0.36 & $\mathbf{0 . 0 2}$
\end{tabular}

Notes: The names of colors are those used by the manufacturer. Responses of bees saturated near contrast values of 0.4 in bright light. The Canson papers were kindly calibrated by MV Srinivasan and SW Zhang. Bold numbers show where contrast between two numbered papers was negligible. random sample from a binomial distribution. An exact $P$-value was computed using a binomial test, without any normal assumption or central limit theorem claim, with an estimate of the bias and a two-sided test to reject the hypothesis that the bias is 0.5 . The test was two sided because values on either side of 0.5 occurred. For example, if $n=62 \%$, then Prob (estimated bias 0.5$)>0.12$. Here the probability is taken with respect to a binomial distribution with bias 0.5 and $n=100$. From a table of $P$-values, $P=0.020,2 \%$, which is acceptable.

Every effort was made to design tests that gave clear yes/no answers. In the most informative tests when the bees failed to find a cue, the missing input could be supplied in a further test. A poor score may mean poor learning of features displayed in the test, little to distinguish in the test patterns, or conflicting effects of opposing inputs.

\section{Setting and illustrations}

In each experiment, it is essential to refer to the corresponding illustration while reading the text. Two training patterns are shown at the top of each illustration. One may be a plain color with no pattern. The rewarded one on the left is marked by $(+)$, followed by pairs of test patterns, with corresponding scores and a histogram. Each illustration should be read from the top down as each successive test is considered, but actually tests were interleaved and most experiments took many days. Conclusions were deduced logically from the test results.

In the previous work, ${ }^{10-20}$ the bees did not learn the whole training patterns and then compare them with the test patterns. Usually, they learned by trial and error; so they remembered their errors and learned to avoid cues in the unrewarded pattern. When they returned for more reward, they recognized the cues to avoid. When they needed to compare two targets, they learned inputs from both. They learned a few simple cues in order of preference, just for the particular training patterns on hand, and on their return, they looked only for the cues they had learned, not for the whole pattern.

\section{Results \\ Bees restricted to green receptor channels measured angular width between vertical edges}

A small group of bees was trained to discriminate between two patterns, each with a single yellow vertical bar $\left(8^{\circ}\right.$ or $16^{\circ}$ wide) in similar locations on a black background (Figure $2 \mathrm{~A}$ ). This was a useful combination of colors that displayed negligible signals to blue receptors and strong green modulation (Table 1) that was the same on each target, leaving little 


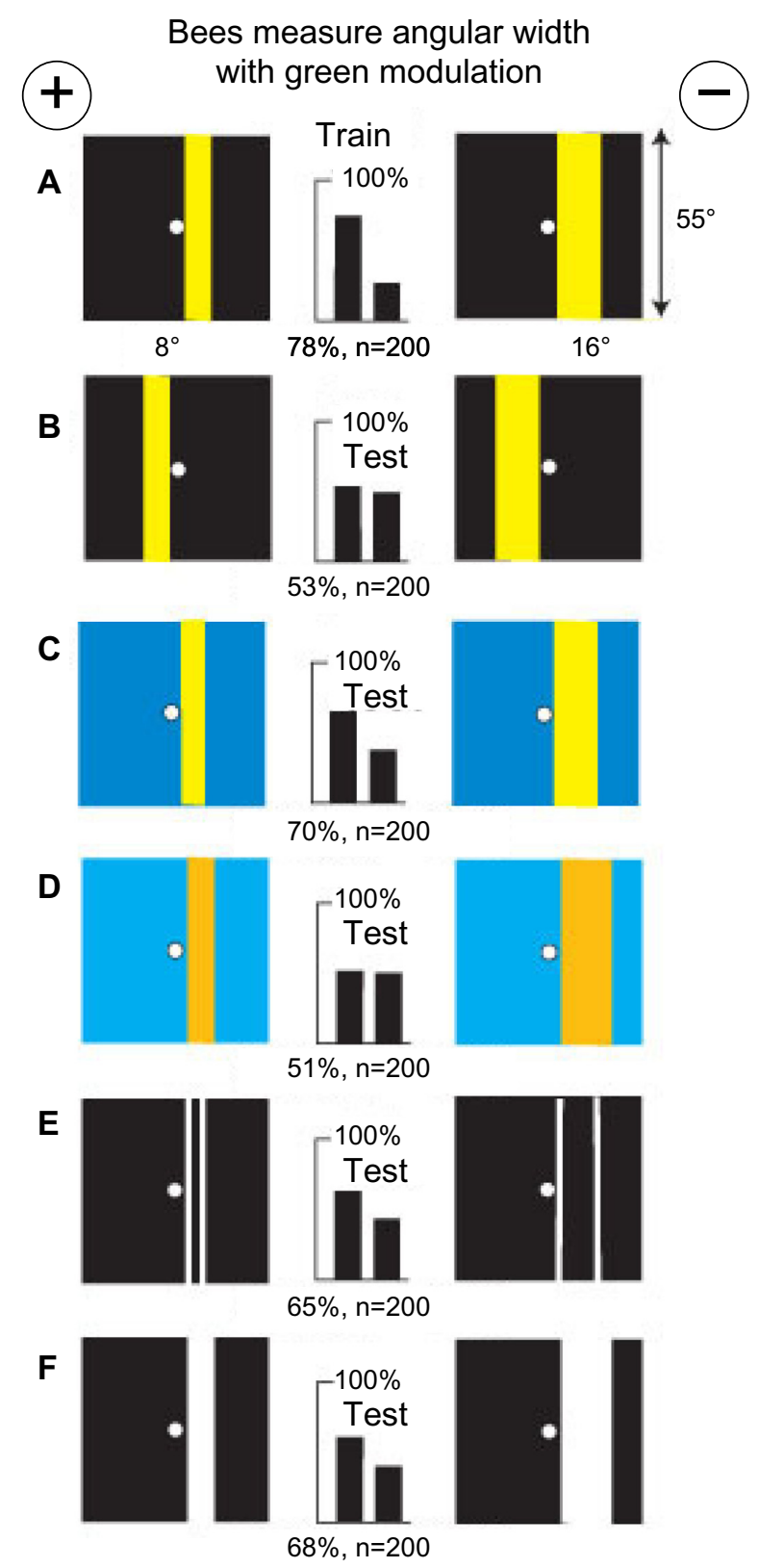

Figure 2 Yellow bars with low blue content and strong green contrast on black backgrounds were distinguished by the width between edges.

Notes: (A) Training patterns with single yellow vertical bars of $8^{\circ}$ and $16^{\circ}$ on black backgrounds. (B) A small shift of the bars to the other side of the reward hole destroys the recognition. (C) The trained bees could distinguish yellow on ultramarine that was equiluminant to blue receptors. (D) With buff on blue, equiluminant to green receptors, recognition failed. (E) The trained bees recognized the difference in separation of thin white vertical lines. (F) White bars increased green contrast but added blue content that reduced the score.

for the bees to distinguish. A small shift of the bars to the other side of the reward hole spoiled all discrimination (Figure 2B), showing that the bars had been strictly located relative to landmarks elsewhere in the apparatus. In a test, trained bees distinguished the same yellow bars when they were equiluminant to blue receptors on an ultramarine background (Figure 2C) because some green contrast was available and they required nothing in the blue receptor channel. With similar bars of buff on blue, with no green contrast, recognition failed because green contrast was essential (Figure 2D). The trained bees recognized a difference in separation of thin white vertical lines in test targets with no color difference (Figure 2E), showing that the training bars were distinguished by width. Plain white bars were also effective (Figure 2F). The factor that could account for the lower scores in Figure 2E and $\mathrm{F}$ was the unexpected increase in blue content.

\section{In patterns with little blue content, bees located a concentration of green modulation and measured angular width}

A small group of bees was trained to discriminate between two patterns, each with three yellow vertical bars ( $4^{\circ}$ wide) on a black background, with the bars more widely separated on the rewarded target (Figure 3A). There was a negligible blue

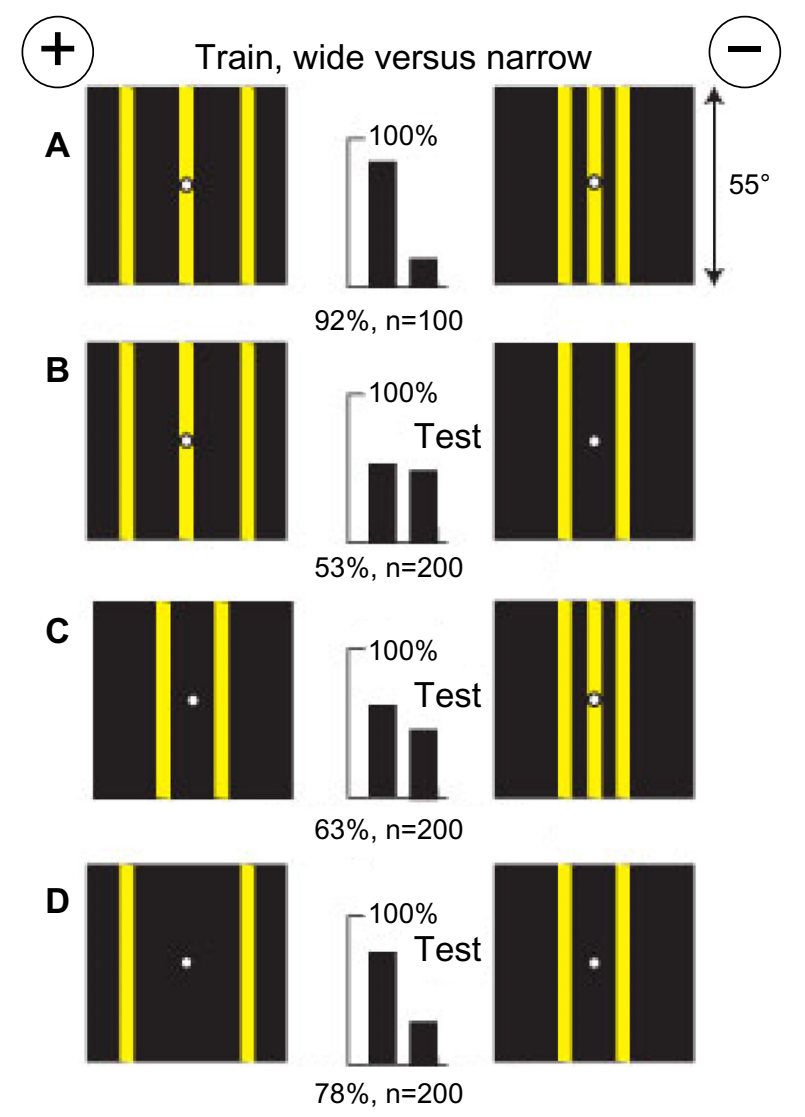

Figure 3 A concentration of green modulation was a stronger cue than a difference in total width.

Notes: (A) Training patterns. (B) Removal of one critical bar showed that the rewarded pattern and total widths in the training were not remembered. (C) With equal widths, the concentration of green modulation of the unrewarded target was recognized and avoided. (D) The difference in width was recognized when the total green modulation was similar and the concentration of edges was removed, illustrating how a forced choice distorts scores. 
content or blue contrast and the same length of edge and green modulation on each target, leaving the bees with little choice. The trained bees failed to distinguish when the central bar was removed from the unrewarded target (Figure 3B), suggesting that they had learned to avoid the three bars generating locally concentrated modulation. In a test with two bars versus three bars with the same total width, the score was much reduced (Figure 3C), showing that the difference in pattern period was not the only cue and suggesting that a difference in width had been learned. With the width between outside edges preserved but only two bars on each target (Figure 3D), the targets were easily distinguished, showing that the difference in total width was a powerful cue.

\section{Gratings with no color difference and no blue contrast were distinguished by a measure of total modulation in the green receptor channel}

A group of bees was easily trained to distinguish a vertical grating of hemp and ultramarine bars with a period of $16^{\circ}$ versus a similar grating with a period of $8^{\circ}$ with each grating filling the target (Figure 4A). These targets were equiluminant for blue receptors (Table 1) and displayed no difference in average or total color. The trained bees had not learned the rewarded training pattern, and in a test, they abandoned it in favor of a larger period (Figure 4B). They had learned to avoid narrow bars. When tested with gratings as in the training, but with an equal number of vertical edges, they were insensitive to the difference in period (Figure 4C), showing that the critical cue was a quantitative measure of the total modulation. When tested with two gratings of buff and blue, equiluminant to the green receptors and with the same periods as the training patterns, they failed (Figure 4D), showing that memory was all in the green channel and could not be transferred to a blue channel. These results are positive evidence that they did not remember the whole patterns or measure the period of the gratings, but learned only to avoid the greater green modulation.

\section{Gratings equiluminant to green receptors and equal in color were distinguished by a measure of total blue modulation}

A group of bees learned to distinguish a vertical grating of buff and blue bars with a period $16^{\circ}$ from a similar grating with a period of $8^{\circ}$ with each grating filling a target (Figure 5A). These targets were equal in total color and contrast, and equiluminant to the green receptors. When offered a target

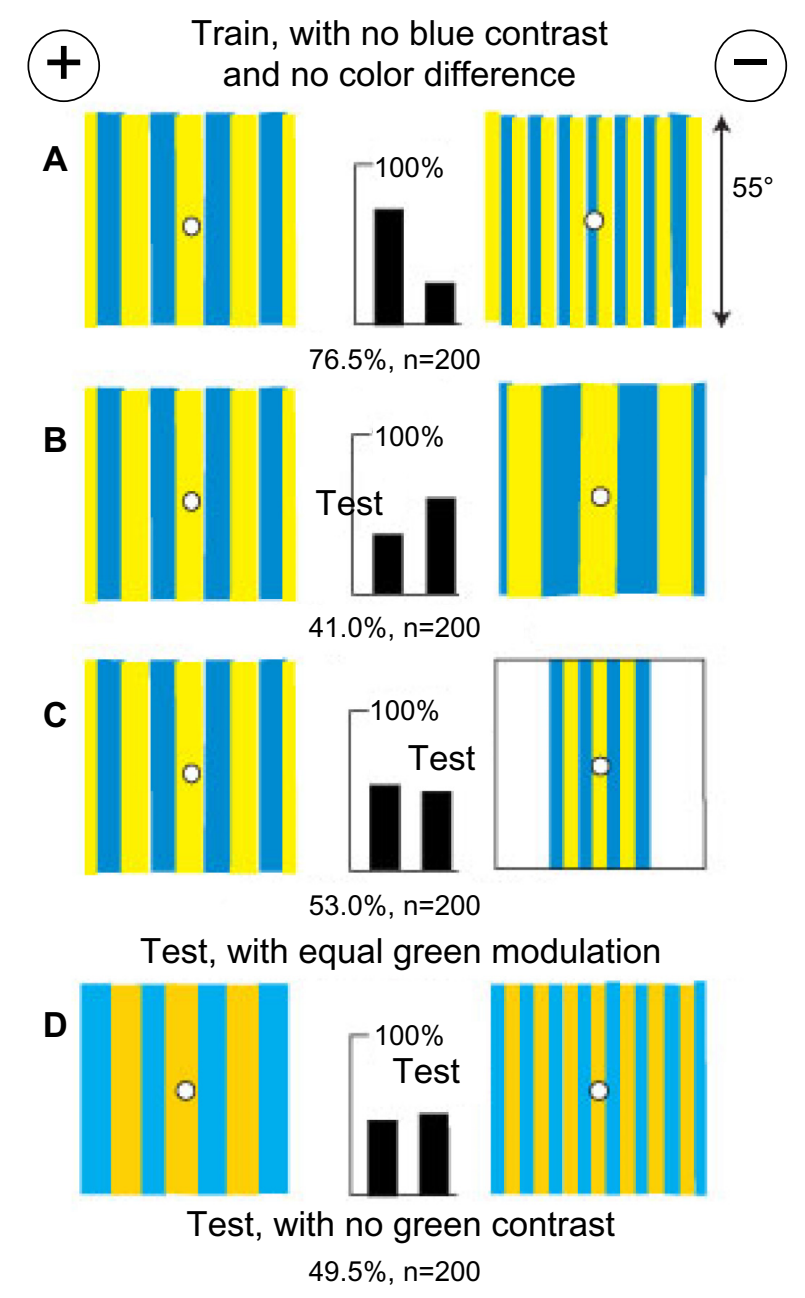

Figure 4 Gratings equiluminant to blue receptors and of equal color and width were distinguished by different amounts of total green modulation, not pattern period. Notes: (A) Training patterns of ultramarine and hemp, equiluminant to blue receptors. (B and $\mathbf{C}$ ) The rewarded target was distinguished from a similar grating with less modulation, but not from one of different width that displayed the same modulation. (D) Gratings equiluminant to the green receptors but otherwise similar to the training targets were not distinguished.

with still larger period, the trained bees avoided the rewarded training target, showing that they had learned to avoid the greater blue modulation, not the particular period (Figure 5B). When the total blue modulation on each was made equal without change in period, the discrimination failed (Figure 5C) showing that the bees had measured a difference in total modulation in the training targets and had not learned the rewarded target or the difference in period. The trained bees avoided greater blue modulation, irrespective of pattern and different blue content (Figure 5D).

A single thin vertical black line added to the rewarded target more than canceled the learned preference (Figure 5E), although the bees had not learned that target in the training. Further experiments, mentioned below, showed that any added green contrast interfered with the use of blue modulation. 
$+$

A

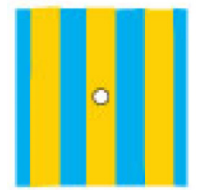

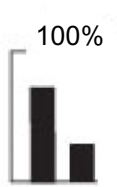

$75.0 \%, n=200$

B

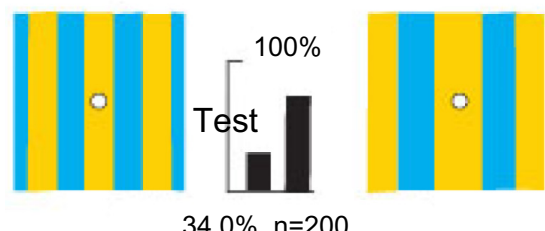

C

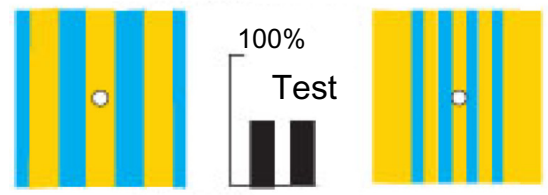

$49.5 \%, n=200$

D

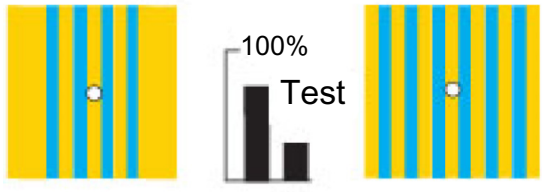

$71.0 \%, n=200$

E

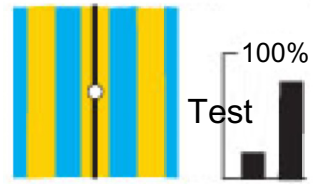

$22 \%, n=100$

$\mathbf{F}$

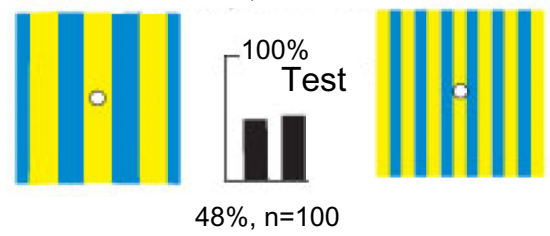

Figure 5 Gratings with equal color content and width, and equiluminant to the green receptors were distinguished by different amounts of total blue modulation, not pattern.

Notes: (A) Training patterns. (B) The trained bees avoided the greater modulation, irrespective of blue content or pattern. (C) With equal blue modulation, the discrimination failed irrespective of period or width. (D) They avoided the greater blue modulation at the same period. (E) A single thin vertical black line more than canceled the learned preference. $(\mathbf{F})$ With similar gratings, but equiluminant to blue receptors, they found no cue.

With targets similar to those in the training but equiluminant to blue receptors (Figure 5F), the trained bees found no cue, showing that they had learned only blue modulation.

\section{Blue modulation was recognized only} in the locations where it was learned and irrespective of reversal of contrast

In the next experiment, the bees were trained to avoid a thin vertical blue bar on a buff background versus a plain buff target, with no green contrast. With a bar $2^{\circ}$ wide, training failed, but a bar $4^{\circ}$ wide with greater blue content was effective (Figure 6A). The bar displayed blue content and blue contrast. In a test when the bar was moved to the other side of the reward hole, the bees failed to respond (Figure 6B),

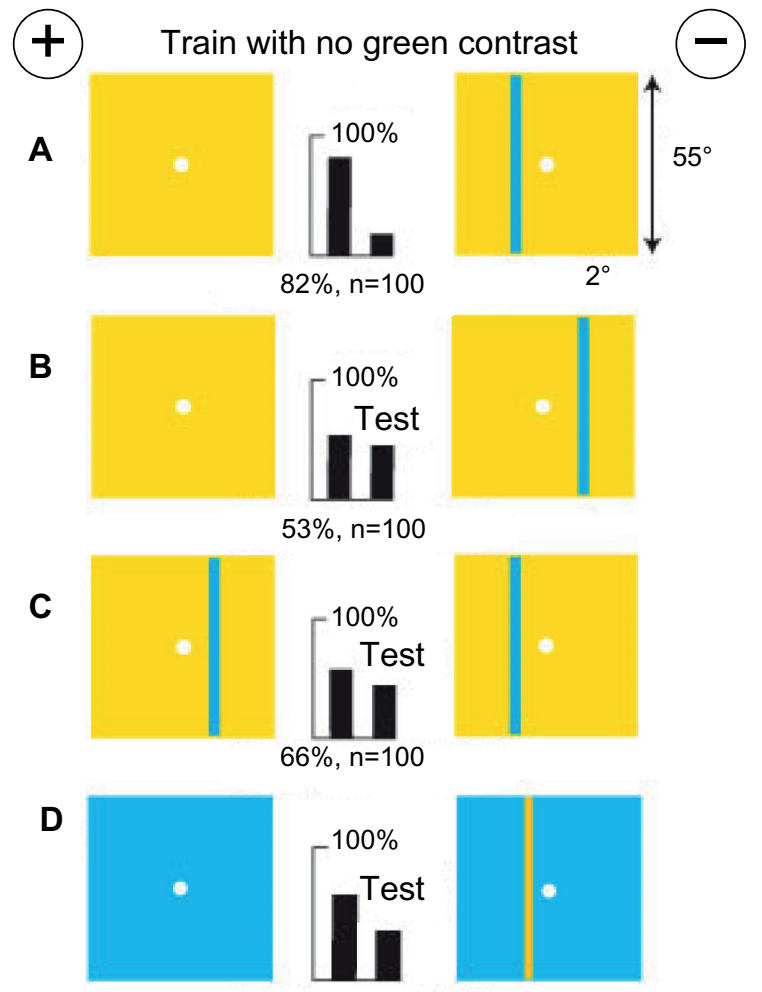

E
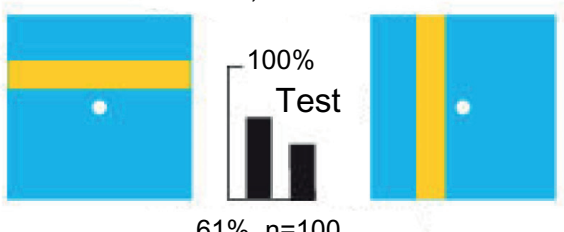

$61 \%, \mathrm{n}=100$

$\mathbf{F}$

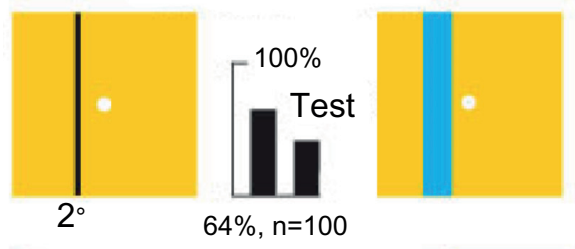

G

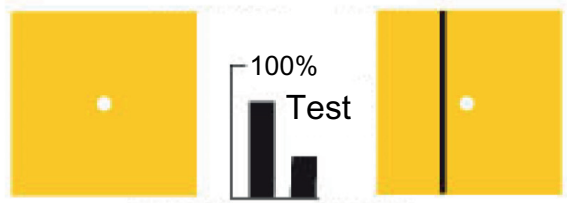

$76 \%, \mathrm{n}=100$

Figure 6 Blue modulation was located irrespective of reversal of contrast and recognized in a thin black line at the expected place.

Notes: (A) Training patterns with a $4^{\circ}$ blue bar on buff, equiluminant to the green receptors. (B) The blue bar was not recognized in a new position. (C) A blue bar was distinguished from a displaced blue bar. (D and $\mathbf{E})$ Similar tests with the colors reversed. (F) The blue bar was distinguished from a similar black bar. (G) Blue modulation was recognized in a black bar at the expected place. 
showing that the memory location was retinotopic and the rewarded target had not been learned. A test with bars on each target showed poor discrimination (Figure 6C).

The trained bees distinguished a buff bar $4^{\circ}$ wide on a blue background from a plain blue target, showing that the expected blue modulation was detected despite the reversal of contrast (Figure 6D). A buff bar $12^{\circ}$ wide on a blue background was scarcely distinguished from the same bar placed horizontally (Figure 6E), showing that a difference in blue content had been learned. A blue bar $12^{\circ}$ wide on a buff background was distinguished from a vertical black line $2^{\circ}$ wide, because the bees had been trained to avoid blue (Figure 6F). Finally, they responded to a thin black vertical line at the expected position of the blue bar in the training (Figure 6G). In all these tests, they showed that they avoided blue or blue modulation at its expected position on the unrewarded training target.

\section{Green modulation canceled blue modulation and bees measured angular width between two vertical bars with blue modulation}

A fresh group of bees was trained to distinguish between a vertical grating of buff on blue from the same grating horizontal with both on a black background. There was no green contrast in the grating and no difference in contrast at the outer edges, but a difference in blue modulation was available. The bees learned rapidly to a high score (Figure 7A). When tested with similar patterns in black on white, the trained bees failed although the blue modulation was greater than in the training (Figure 7B). Failure was caused by the appearance of strong green contrast, which canceled the cue that the bees had learned, and perhaps also by flooding the targets with a strong blue content.

A fresh group of bees was trained to distinguish two widely spaced vertical blue bars on a buff background from the same bars placed close together (Figure 7C). These targets conveyed nothing when the bars were horizontal (Figure 7D). The difference in separation was distinguished with black bars on gray ( $40 \%$ black) despite the introduction of green contrast (Figure 7E). The cue was not known, but forced choice always magnified the effect of a small residual difference. The training targets were equiluminant to the green receptors; therefore, the trained bees looked for blue modulation. However, the addition of a greater green modulation with a single black vertical bar at the center of each training target destroyed the discrimination (Figure 7F).

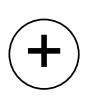

Train, with no green contrast and no blue difference

\section{A}
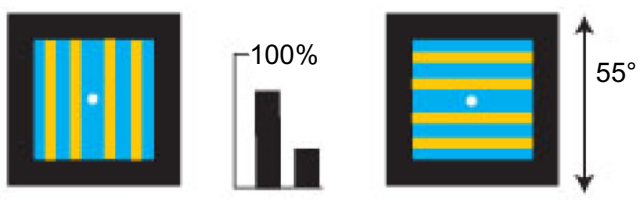

$88.5 \%, n=200$

\section{B}

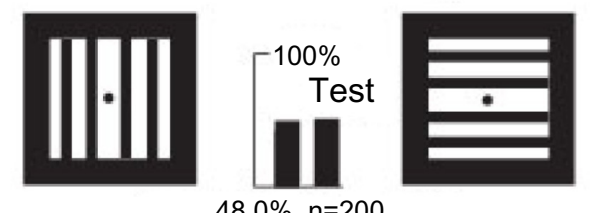

$48.0 \%, n=200$

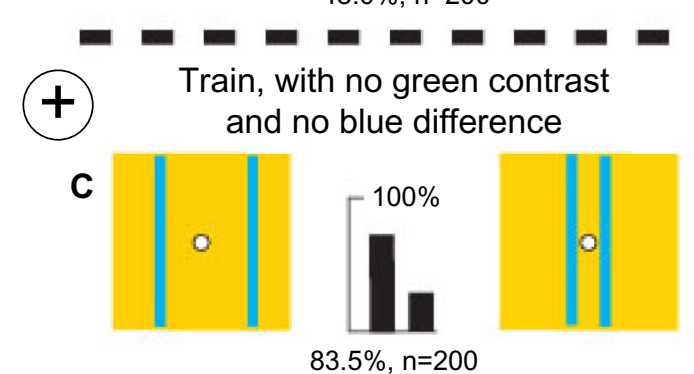

D

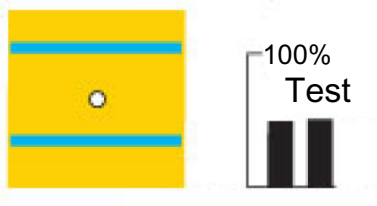

$49.0 \%, n=200$

E
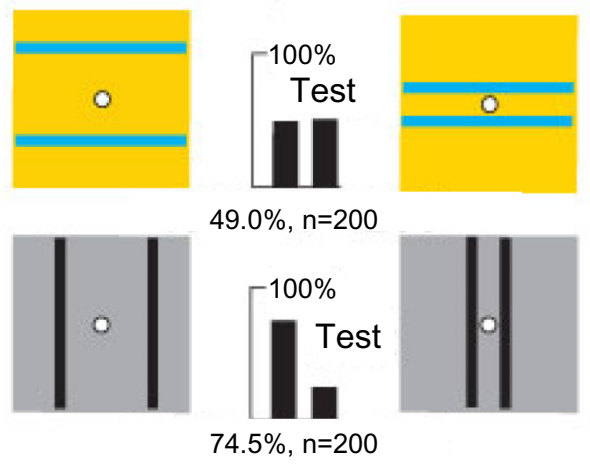

$74.5 \%, n=200$
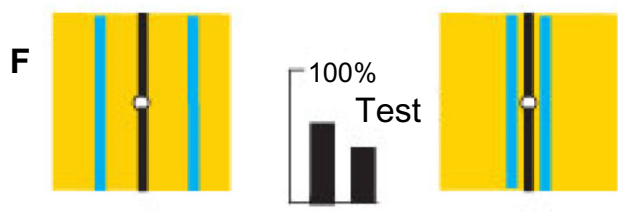

$60.5 \%, n=200$

Figure 7 Green modulation canceled blue modulation and bees measured angular width between two vertical bars with blue modulation.

Notes: (A) Training patterns with a difference in blue modulation but no green contrast or color difference. (B) Failure with similar patterns in black on white, because strong green contrast canceled blue modulation. (C) New training patterns. (D) The rotated patterns were not recognized. (E) The separation was readily distinguished with black bars on gray (60\% white). (F) Addition of a black vertical bar destroyed the discrimination.

\section{Blue modulation was sensitive, located in position, and robust}

In this example, the bees were trained to distinguish a vertical buff bar $10^{\circ}$ wide on a blue background, with no green contrast, from a plain blue target (Figure 8A). The trained bees could easily distinguish a bar only $2^{\circ}$ wide versus a 


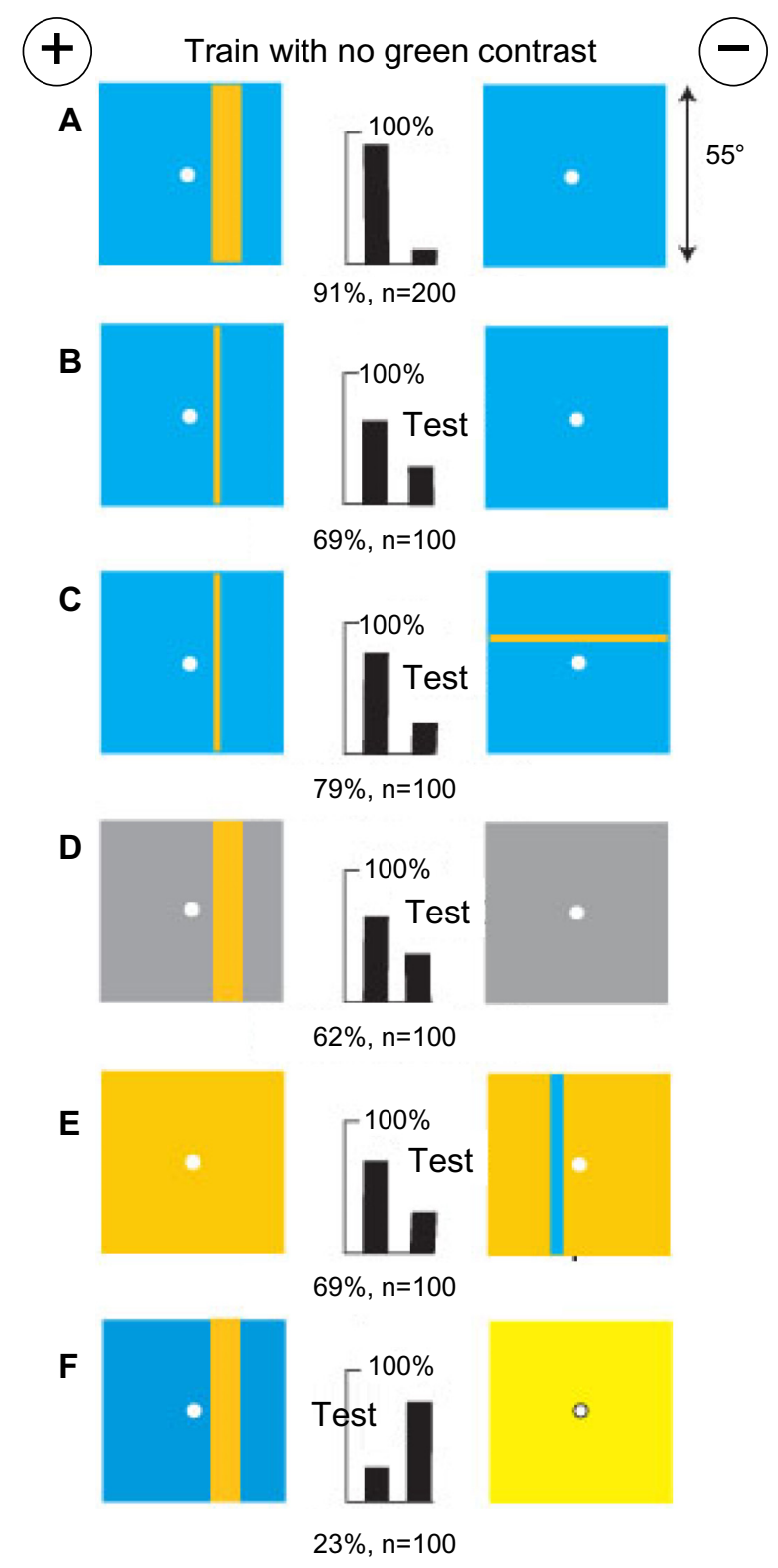

Figure 8 Blue modulation and blue content were learned simultaneously but separately.

Notes: (A)A broad buff bar on blue displayed a width, a difference in blue modulation and blue content, but not in green modulation. (B)A thin bar was less effective because of less blue difference. (C)With equal blue content, blue modulation was still available. (D) A gray background (60\% white) had blue content but generated green modulation that reduced the response. (E) The greater blue content in a blue line on buff was avoided because was stronger than the blue modulation. (F) Yellow was preferred to the rewarded target because the bees were trained to avoid most blue.

plain background (Figure $8 \mathrm{~B}$ ) or versus a horizontal $2^{\circ}$ bar (Figure 8C). They had detected the difference in modulation by scanning and not orientation because they could not learn orientation with the blue modulation pathway. ${ }^{12}$ They also detected a buff bar on a gray background ( $60 \%$ white) versus a plain gray target (Figure 8D) because gray displayed some blue contrast and blue content. When tested with a plain buff target versus a $4^{\circ}$ blue bar on a buff background, with no green contrast (Figure 8E), the trained bees avoided the greater blue content. They ignored the blue contrast because the bar had been moved. When tested versus yellow, they avoided blue (Figure $8 \mathrm{~F}$ ), showing again that in the training, they had learned to avoid the greater blue content as well as blue modulation.

\section{With no green contrast, bees measured blue modulation, angular width, and amount of blue}

A fresh group of bees was trained to distinguish a vertical buff bar $\left(8^{\circ} \times 55^{\circ}\right)$ on a blue background from a plain blue target (Figure 9A). The trained bees failed to distinguish the training bar from a buff rectangle of the same area $16^{\circ} \times 27.5^{\circ}$ in a similar position (Figure $9 \mathrm{~B}$ ), but detected a difference with a buff rectangle of $20^{\circ} \times 22^{\circ}$ (Figure 9C). These test rectangles had the same area as the bar and were centered at the same place with no difference in color or blue content. The result suggests that bees were distinguished by a difference in the widths of bars or lengths and positions of vertical edges.

The trained bees easily distinguished the training bar from two thin vertical bars, each $2^{\circ}$, separated by $4^{\circ}$ (Figure 9D), and very easily from four bars, each $2^{\circ}$, separated by $2^{\circ}$ (Figure $9 \mathrm{E}$ ). In each test pair, the amount of blue was similar on the two targets, and some of the vertical edges were in the expected positions. The results show that thin $2^{\circ}$ bars were resolved by blue modulation and that the bees preferred the expected width and measure of the bar in the training. On the other hand, a single bar $16^{\circ}$ wide was not distinguished because the difference in blue content was a stronger cue than bar width (Figure 9F). In this example and in Figures 6 and 8, one of the training targets had no pattern and the bees detected the same features as in experiments with two patterns, but unlike Figure 6, here they learned the rewarded target.

\section{With a bar on each target and no green contrast, bees learned their angular widths and avoided unexpected extra modulation}

Next, a group of bees was trained to distinguish a buff bar $6^{\circ}$ wide from a similar bar $12^{\circ}$ wide, both on blue backgrounds (Figure 10A). The trained bees failed to distinguish between a $16^{\circ}$ bar and a $12^{\circ}$ bar (Figure 10B) and had difficulty with a $6^{\circ}$ bar and an $8^{\circ}$ bar (Figure 10C), all on the same blue 


\section{(† Train with no green contrast}

A

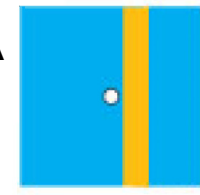

$8^{\circ}$

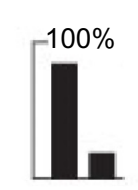

$88 \%, n=100$

B

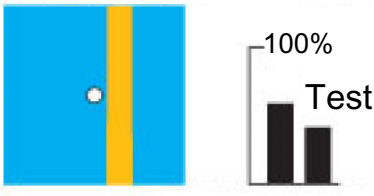

$59 \%, n=100$

C

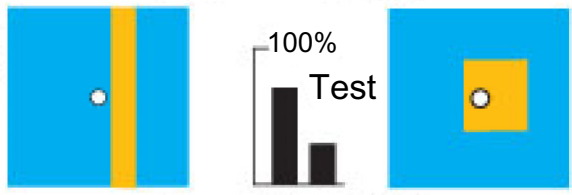

$77 \%, n=100$

D

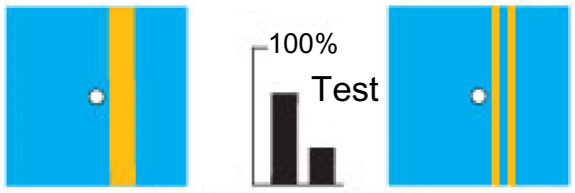

$73 \%, n=100$

E

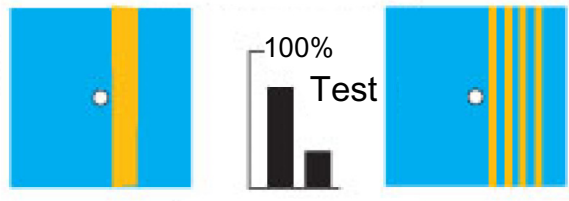

$83 \%, n=100$

$\mathbf{F}$
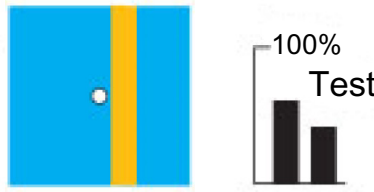

$57 \%, n=100$

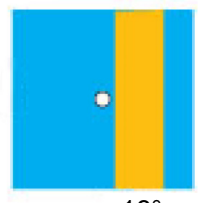

$16^{\circ}$

Figure 9 In the absence of green contrast, a measure of blue modulation was preferred over bar width or amount of blue.

Notes: (A) A buff bar $8^{\circ}$ wide on blue displays a difference in blue modulation and blue content but no green modulation. (B) A buff rectangle $8^{\circ} \times 13.5^{\circ}$, of equal area to the training bar, displayed blue modulation that was not distinguished from the training pattern. (C) A buff rectangle $10^{\circ} \times 10.8^{\circ}$, also of equal area, was easily distinguished. (D and E) Two bars $2^{\circ}$ wide, $4^{\circ}$ wide apart, or four bars $2^{\circ}$ wide, $2^{\circ}$ wide apart, were also easily distinguished. (F) A single bar $16^{\circ}$ wide, twice the area of the training bar but displaying similar blue modulation, was not distinguished.

background, showing that whatever had been learned was accurately measured. Tests with each training target versus plain blue showed that something had been learned from each (not illustrated). In a test with thin buff bars $2^{\circ}$ wide replacing the edges of the training bars, the trained bees distinguished very well, showing that an accurate location of the blue modulation at both edges of at least one bar was a preferred input (Figure 10D). In a test with a $6^{\circ}$ bar versus a pair of edges of the same width (Figure 10E) or with one
Train, with no green contrast, equal blue modulation, and different bar widths

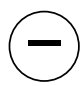

A

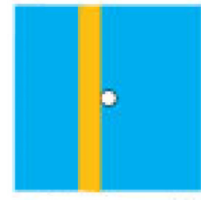

$6^{\circ}$

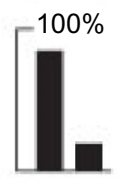

$89 \%, n=100$

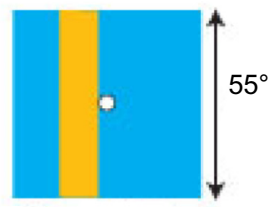

$12^{\circ}$

B

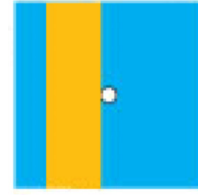

$16^{\circ}$

C

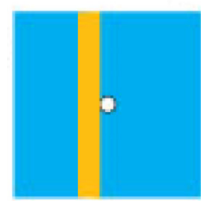

$6^{\circ}$

D
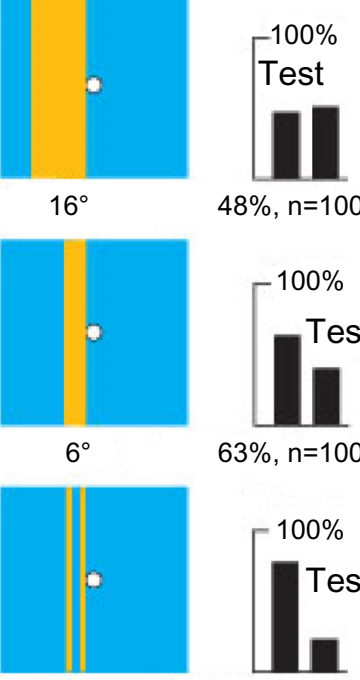

$48 \%, n=100$
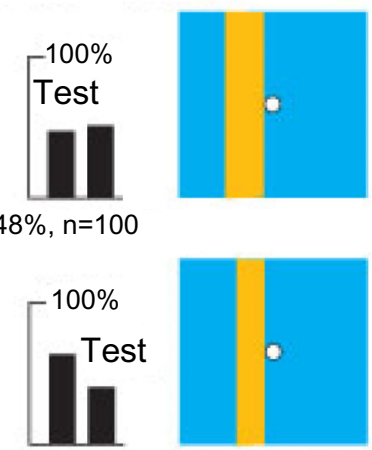

$63 \%, n=100$

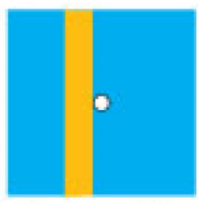

$8^{\circ}$
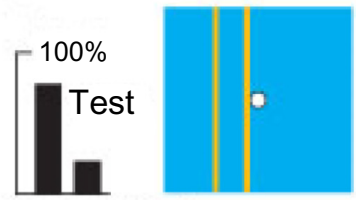

$80 \%, n=100$

E
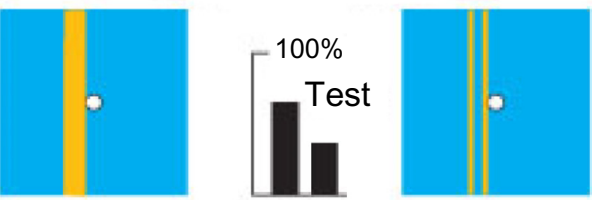

$69 \%, n=100$

$\mathbf{F}$
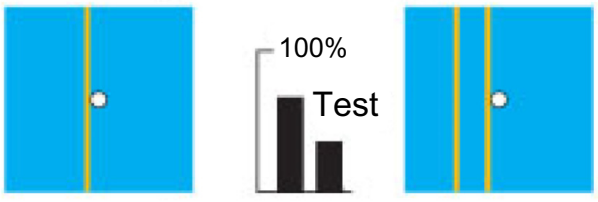

$66 \%, n=100$

Figure 10 Small differences of blue content were measured and widths of two bars were compared using blue modulation on both targets.

Notes: (A) Training patterns, equiluminant for green receptors. (B) The difference in width canceled a difference in the blue content. (C) The trained bees went toward more blue and an expected width. (D)Widths between lines of blue modulation were compared. (E and F) Uncertain, probably there were conflicting cues, blue content, width between blue modulation lines, and unexpected extra blue modulation.

bar versus two bars (Figure 10F), the trained bees avoided the extra modulation, and probably, they were also attracted to the target with more blue content.

To distinguish the bars in this experiment, the bees located two positions of blue modulation. Intrinsic to the eye anatomy, they had the angle between these inputs available. They remembered the difference between $6^{\circ}$ and $12^{\circ}$ edge separations with blue modulation when green modulation was lacking. 


\section{Bees trained on black/white gratings with no difference in total color or width learned only a difference in green modulation}

A fresh group of bees was trained to distinguish between two black/white gratings that displayed equal areas of white and therefore equal blue content, but the rewarded target with three black bars had less than half the length of vertical edge than the other with seven bars (Figure 11A). The trained

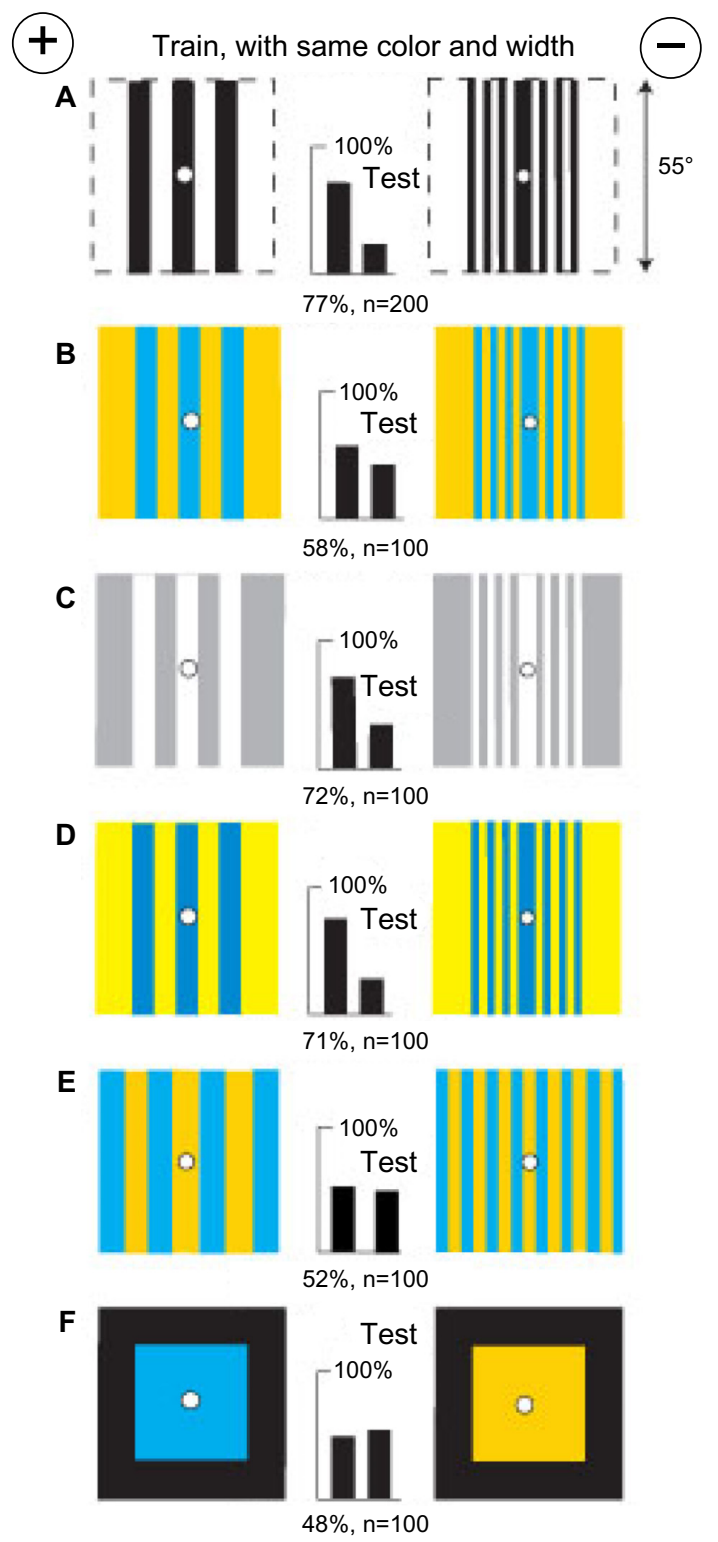

Figure II Black and white patterns of equal blue content and contrast were distinguished by a green modulation difference.

Notes: (A) Training patterns. (B) Negligible response to the same patterns with no green contrast. (C) Good response to the same patterns in white on gray, irrespective of contrast reversal. (D) Excellent response to the same patterns equiluminant to the blue receptors. (E) No discrimination between plain gratings equiluminant to the green receptors. (F) No color preference between blue and buff with equal green contrast on black. bees performed badly when tested with the same gratings that were blue on buff and equiluminant for green receptors (Figure 11B), but easily distinguished white bars on gray (Figure 11C) or ultramarine bars on hemp (Figure 11D), both of which displayed abundant green contrast. They failed when tested with regular gratings with no green contrast (Figure 11E) or with plain colors blue and buff on black backgrounds (Figure 11F), showing that in the training, learning of blue modulation had been inhibited by the strong blue and green signals. Colors were of no interest because the training patterns were equal in blue content.

\section{Bees trained on blue-on-buff gratings with no difference in total width or color and no green contrast learned only a difference in blue modulation}

A fresh group of bees was trained to distinguish between two gratings of blue vertical bars on buff that displayed no green contrast and equal width and blue content. The rewarded target with three blue bars had less than half the total length of vertical edge displayed by the other with seven narrower bars (Figure 12A). The trained bees responded with a reduced score to black bars on white (Figure 12B) and also to white bars on gray (Figure 12C) but succeeded with a similar set of bars of ultramarine on hemp that displayed no blue difference (Figure 12D). Therefore, they had learned the blue modulation difference, as confirmed when they failed with gratings that differed only in blue modulation (Figure 12E). When tested with a blue square on black versus a hemp square on black, with no green difference, they avoided the greater blue modulation at the vertical edges between blue and black (Figure 12F). That was all they had learned because the training patterns displayed no color difference and no green contrast.

\section{Memory of location played a large part when discrimination of mirror images was limited to green modulation alone}

Earlier studies by many authors ${ }^{10}$ showed that bees discriminated bilaterally symmetrical black/white patterns from similar asymmetrical patterns if one of the patterns had a vertical axis of symmetry. They even discriminated when different patterns with a similar orientation of the axis were shuffled, as if the bees detected an abstract symmetry irrespective of pattern. These results remind us that a local concentration of modulation acts like an abstract generalized feature $^{6}$ (Figure 3), but tests for this cue were never made with 


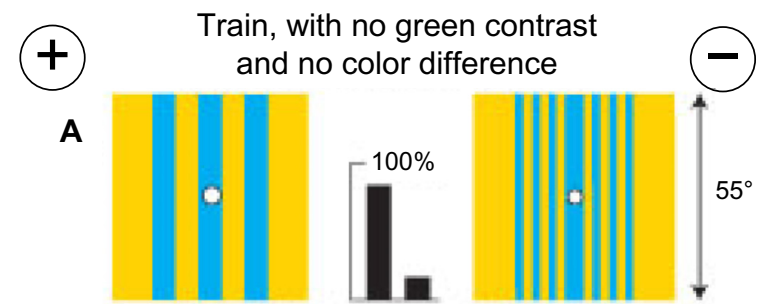

$89.5 \%, n=200$

B

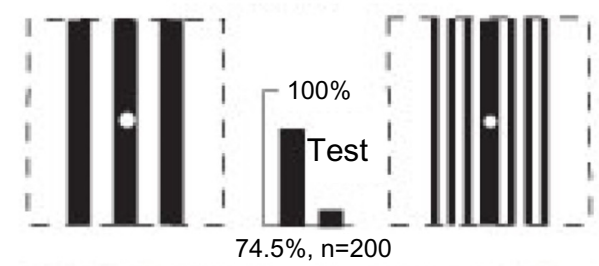

C

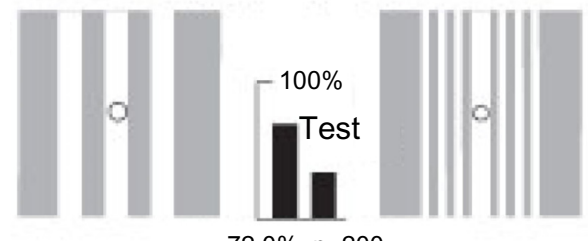

$72.0 \%, n=200$

D

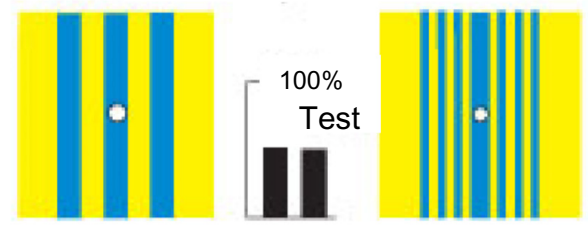

$51.0 \%, n=200$

E

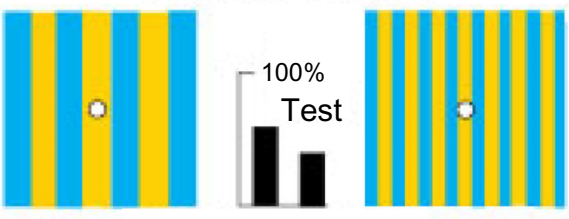

$63.5 \%, n=200$

$\mathbf{F}$

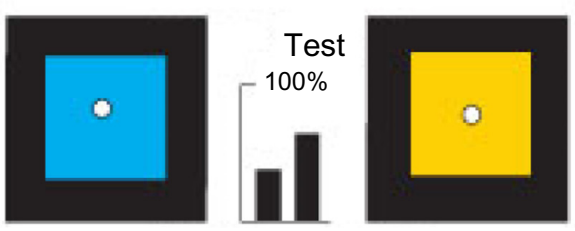

$36.0 \%, n=200$

Figure 12 Gratings with equal blue contrast and blue content but no green contrast were discriminated by a difference in blue modulation.

Notes: (A) Training patterns; bees must learn the difference in blue modulation. (B) The bees recognized the familiar blue modulation in black/white gratings. (C) White and gray gratings were correctly distinguished, irrespective of contrast reversal. (D) Failure with the same patterns equiluminant to the blue receptors. (E) Discrimination between plain gratings with no green contrast by the difference in blue modulation. (F) Avoidance of greater blue modulation with targets of blue and buff on black (with equal green contrast).

asymmetrical patterns. A previous paper on the discrimination of mirror images displaying two colors ${ }^{17}$ showed that the cue was the position of blue separated in the horizontal direction by up to $50^{\circ}$ relative to a vertical edge with green or blue contrast. The angle between was also measured. ${ }^{17}$ Patterns of vertical bars of only a single color also display polarity in the horizontal direction, but many tests were required to discover what the bees detected.

With targets of vertical yellow bars on a black background that displayed insignificant blue differences but abundant green contrast, bees learned several cues (Figures 2 and 3). They detected, measured, and located retinotopic positions of single vertical bars, widths of single bars on each target, the place where the bars were close together, and the absolute angular width between the outside edges of a group of vertical bars. With only green modulation, they learned two position cues in each task (Figures 2 and 3).

To analyze further the detection of polarity, a new group of bees was trained to discriminate a pattern with a single yellow bar $4^{\circ}$ wide on the left and a group of three similar bars on the right from the mirror image of the same (Figure 13A). These patterns were readily discriminated although total modulation and widths between outside edges were identical. When the rewarded target was tested versus plain black, the trained bees avoided black, and when black was tested versus the unrewarded target, they tried to avoid both targets and failed (not illustrated). No conclusions can be drawn from their avoidance of black.

Tested with a symmetrical pattern of four bars versus the unrewarded target, the trained bees showed that they had learned the latter (Figure 13B), and when tested with the rewarded target versus the symmetrical pattern, they also showed some recognition (Figure 13C) showing that they had learned something from each training target. The trained bees detected no difference between four bars and one bar (Figure 13D), so had not learned a measure of modulation, but they just managed to recognize the asymmetry with single bars in different positions (Figure 13E). The positions must have been learned relative to vertical edges inside the apparatus. Little can be said about these results until more patterns have been examined.

As a variation of the above, a new group of bees was trained with three yellow bars on the left of the target versus three bars on the right (Figure 13F). As before, the bees learned something from each target, but more from the unrewarded one (Figure 13G and $\mathrm{H}$ ). When tested with the targets in Figure 13A, they failed completely, showing that the position of the three bars was obscured by an additional bar (Figure 13J). They could not distinguish three bars close together from three bars far apart when all were placed symmetrically, showing that they had located the dense region of modulation in relation to landmarks in the apparatus. The preference was for the position and measure of modulation, not abstract symmetry or pattern. 


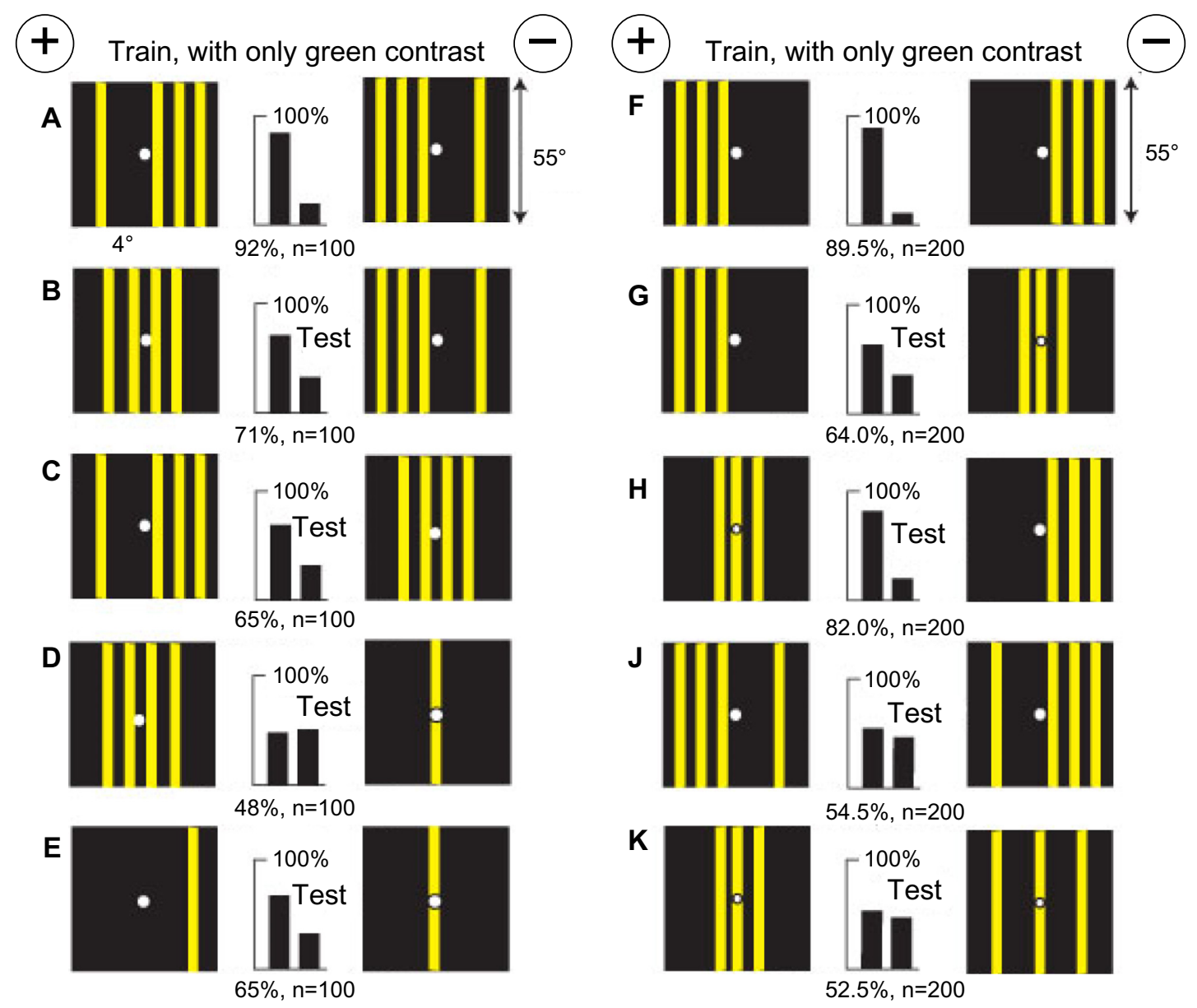

Figure 13 Locations of green modulation were sufficient to distinguish mirror images of vertical bars with negligible blue content.

Notes: (A) Easy discrimination with four yellow bars $2^{\circ}$ wide placed asymmetrically. (B and C) Both training targets were distinguished versus a symmetrical pattern. (D) There was no memory of total modulation or number of bars. (E) Weak detection of asymmetry. (F-K) Similar targets with only three bars. (F) Training patterns. $(\mathbf{G}$ and $\mathbf{H})$ Moving the modulation concentration on the unrewarded target reduced the score, but not on the rewarded target. (J) There was no generalized recognition of polarity. (K) All cues had been moved.

\section{Asymmetrical spacing of four vertical bars was discriminated from symmetrical spacing of the same bars}

A new group of bees was trained to discriminate an asymmetrical pattern of a group of three bars on the left and a single yellow bar on the right from a pattern of four symmetrically arranged bars (Figure 14A). As before, the bars were $4^{\circ}$ wide and total modulation (green only) and widths between outside edges of the patterns were identical. When each target was tested versus plain black (not illustrated), the trained bees avoided black in each case, suggesting nothing because they may have simply avoided black. A test with the rewarded pattern versus its mirror image failed, suggesting that the rewarded pattern as a whole or the polarity had not been learned (Figure 14B).

When tested with four symmetrical bars placed close together in the center versus the same bars spread out, there was little attraction for either target (Figure 14C), showing that the unrewarded pattern as a whole had not been learned either. When the concentration of three bars on the rewarded target was replaced by a single thick bar (Figure 14D), the trained bees failed, showing that three bars were essential and confirming that the unrewarded target or its symmetry had not been learned. The rewarded target was not discriminated from six bars placed symmetrically (Figure 14E), because the expected group of three bars occurred on both targets, but two bars (Figure 14F) or three bars in a group (Figure 14G) placed asymmetrically were distinguished quite well. This experiment showed that a cue, a concentration of modulation placed asymmetrically, was located and measured. Previous work with black and white patterns $^{6}$ and above (Figure 3 ) also showed that the position of an area of extra-dense modulation was the preferred cue.

Finally, a new group of bees was trained with the patterns of Figure 14A interchanged (Figure 14H). As we have seen many times, they did not learn the rewarded pattern 
(+ Train, with only green contrast

A

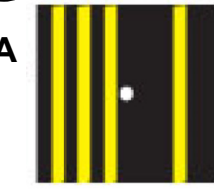

$4^{\circ}$

B

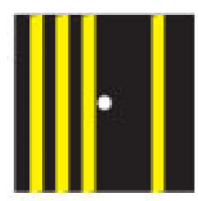

C

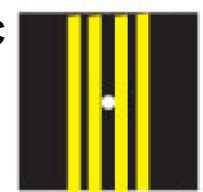

$52 \%, n=100$
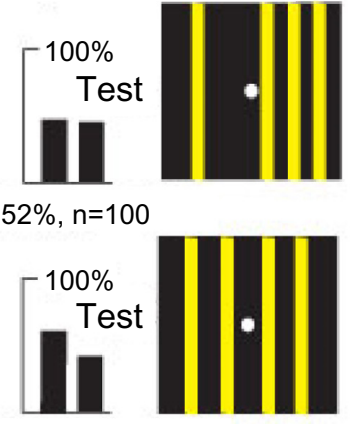

$57 \%, n=100$
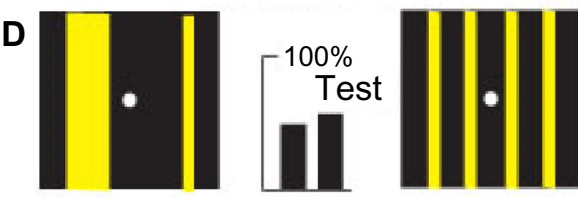

E

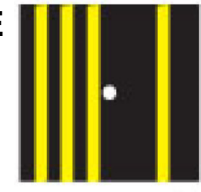

$48 \%, n=100$

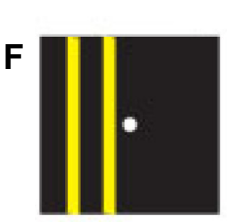

G

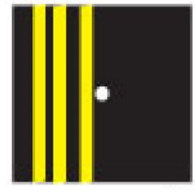

${ }_{55 \%, n=100}^{\text {Test }}$
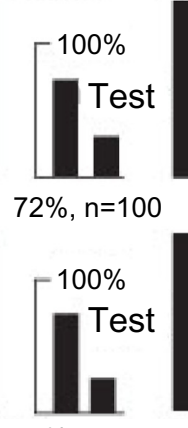

$76 \%, n=100$
$72 \%, n=100$
(-)

$+$

Train, with only green contrast

H
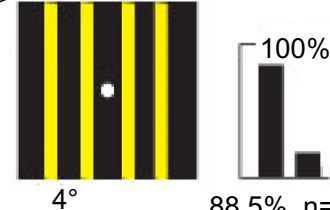

$88.5 \%, n=200$

J

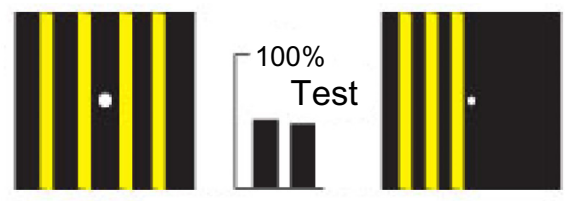

$51 \%, n=100$

K

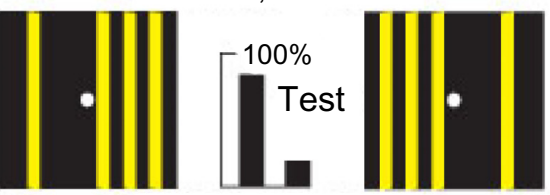

$81 \%, n=100$

L

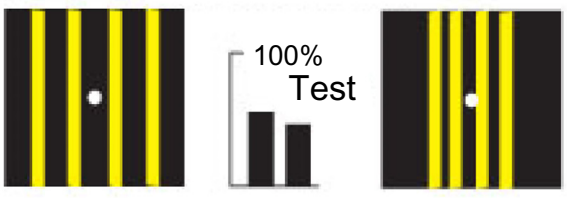

$54 \%, n=100$

M

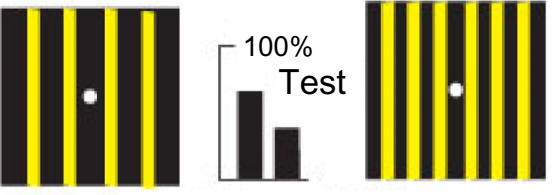

$70 \%, n=100$

N
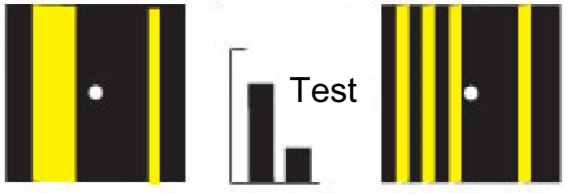

$74 \%, n=100$

P

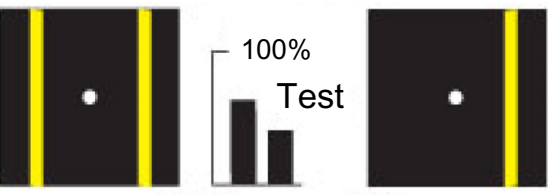

$65 \%, n=100$

Figure 14 Asymmetrical spacing of four vertical bars was discriminated from symmetrical spacing of the same bars.

Notes: (A) Training patterns. (B) No abstract detection of polarity. (B and C) Either training pattern alone was insufficient. (D-G) The concentration of modulation on the left of the rewarded target was the cue. (H-P) Symmetrical spacing of four vertical bars was discriminated from asymmetrical spacing of the same bars. $(\mathbf{H})$ Training patterns. (J) The rewarded pattern was not recognized, and the three bars together lacked the essential fourth bar. (K) The unrewarded target was recognized. (L) The rewarded target was not recognized. ( $\mathbf{M}$ and $\mathbf{N}$ ) Sufficient coincidences of bar locations for recognition of the unrewarded target. (P) Weak recognition with three bars in critical retinotopic positions (compare Figure I3E).

(Figure 14J) but avoided the unrewarded target (Figure 14K). They failed to recognize a concentration of modulation in the wrong place (Figure 14L) and avoided a concentration of modulation in the expected place (Figure 14M and N). With a reduced number of bars in the expected locations, they managed a weak recognition (Figure 14P).

The above set of tests (Figures 13 and 14) showed that when faced with a polarity difference, bees located and measured the strongest signal, a concentration of green modulation, and compared it to the target where modulation was less concentrated in that position, irrespective of which target displayed symmetry or polarity. A concentration of modulation was also a strongly preferred cue in previous work with black and white patterns. ${ }^{6}$ There was no evidence of a generalized recognition of symmetry or asymmetry. 


\section{Effect of different gray levels in the background}

A new group of bees was trained to discriminate a blue square $18^{\circ} \times 18^{\circ}$ on a gray background of $40 \%$ white versus a plain gray background of $40 \%$ white (Figure 15A). It must be remembered that the blue square replaced part of an area of gray. From values of stimulus intensities (Table 1), we see that the rewarded target displayed more total blue content than the

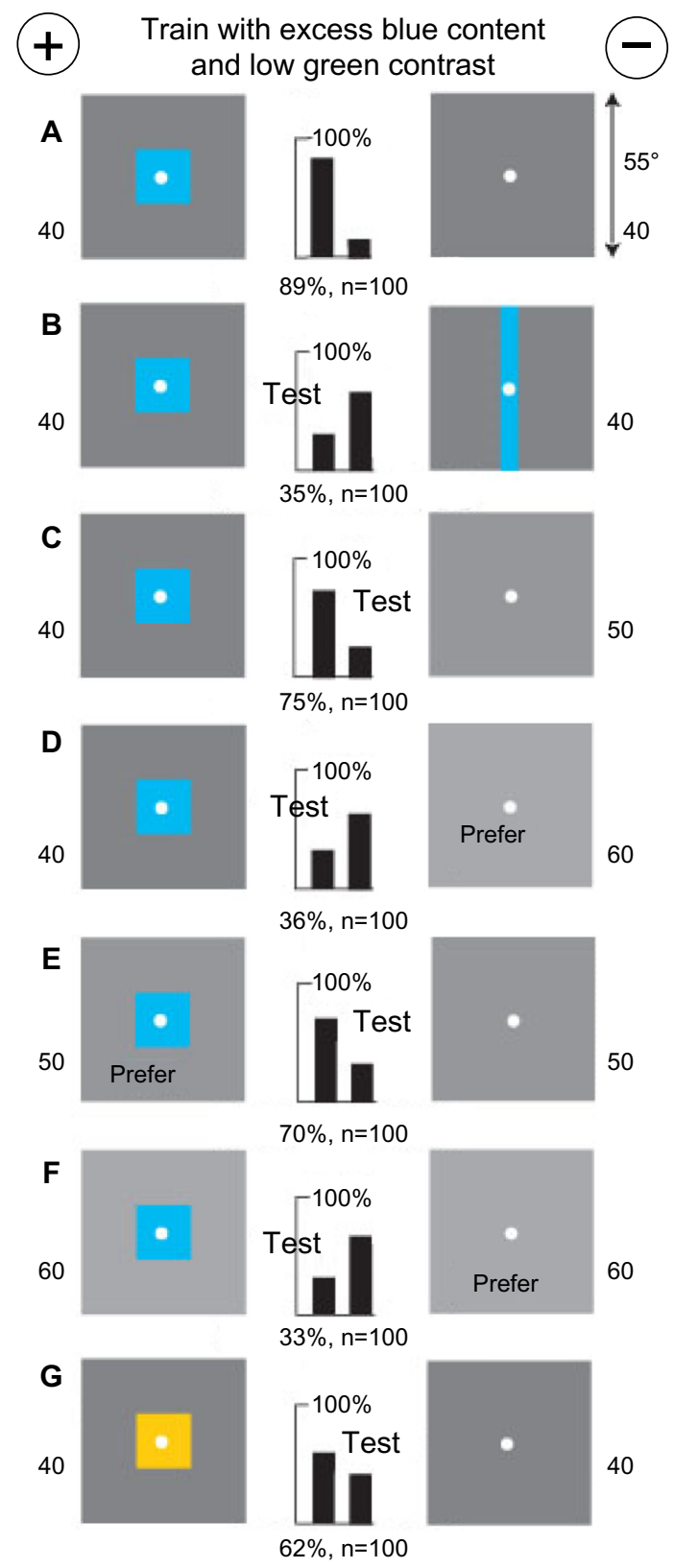

Figure 15 A blue square on a gray background ( $40 \%$ white), versus a plain gray target (40\% white), was detected by greater blue content and some green contrast. Notes: (A) Training patterns. (B) The trained bees preferred a blue bar with greater green modulation (longer edges). (C) Versus $50 \%$ white on the unrewarded target, no change. (D) Reversal of preference versus $60 \%$ white. (E) Blue on $50 \%$ white versus $50 \%$ white resembled the training situation. (F) Blue on $60 \%$ white versus $60 \%$ white reversed the preference. $(\mathbf{G})$ Buff in place of blue in the training patterns removes blue but leaves green contrast unchanged. To match the colored papers, gray levels were indicated by the percentage of white, not black, as indicated by numbers at the sides. other target and a low contrast to the green and blue receptors. In a test with the rewarded target versus a vertical blue bar of the same area of blue, also on a background of $40 \%$ white, the trained bees preferred the greater modulation in the greater length of vertical edge (Figure 15B). Tested with the rewarded target versus a plain gray background of $50 \%$ white (Figure 15C), they discriminated well, but versus a plain gray background of $60 \%$ white, they reversed their preference, because the lighter gray target displayed greater blue content (Figure 15D). With a blue square on a background of $50 \%$ white versus plain gray of $50 \%$ white (Figure $15 \mathrm{E}$ ), they preferred the blue square, but with a blue square on a background of $60 \%$ white versus plain gray of $60 \%$ white (Figure 15F), they again preferred the plain target with the greater display of blue. Tested with a buff square (Figure 15G) instead of the blue square in the training, blue content and blue contrast were reduced but the green modulation was unchanged.

During these changes in the preference, from Figure 15C to $\mathrm{D}$, then back to Figure $15 \mathrm{E}$, and then reverse again to Figure $15 \mathrm{~F}$, the blue square was constant. However, the whole target changed from less blue content to more blue, then less blue, and then back again to more blue. The choices of the trained bees were controlled by the blue content of the background, not by a preference for the blue square. Exactly how many choices were governed by modulation besides blue content was unknown because there were no relevant tests.

\section{One blue square with no blue difference was detected by green modulation}

A new group of bees was trained to discriminate a blue square $18^{\circ} \times 18^{\circ}$ on a gray background of $55 \%$ white versus a plain gray target of 55\% white (Figure 16A). This gray level is equiluminant with the blue square, which was therefore invisible to blue receptors, leaving green modulation as the only available input. Accordingly, when the blue square on various background levels of gray was tested with plain gray targets of similar gray levels (Figure 16B-E), the blue square was always preferred because it consistently displayed edges with green contrast and the polarity of contrast was not detected. The blue square on $55 \%$ white from the training was distinguished from the same blue square on $40 \%$ white with the latter displaying less green modulation (Figure 16F). The color of the square had not been learned in the training because it had not been detected as the first preference. Similarly, when the blue square in the training target was replaced by buff, with similar green contrast, the trained bees were able to distinguish it by its green contrast (Figure 16G). In this example, the bees learned nothing via the blue receptor pathways although the square was blue and on the rewarded target. 


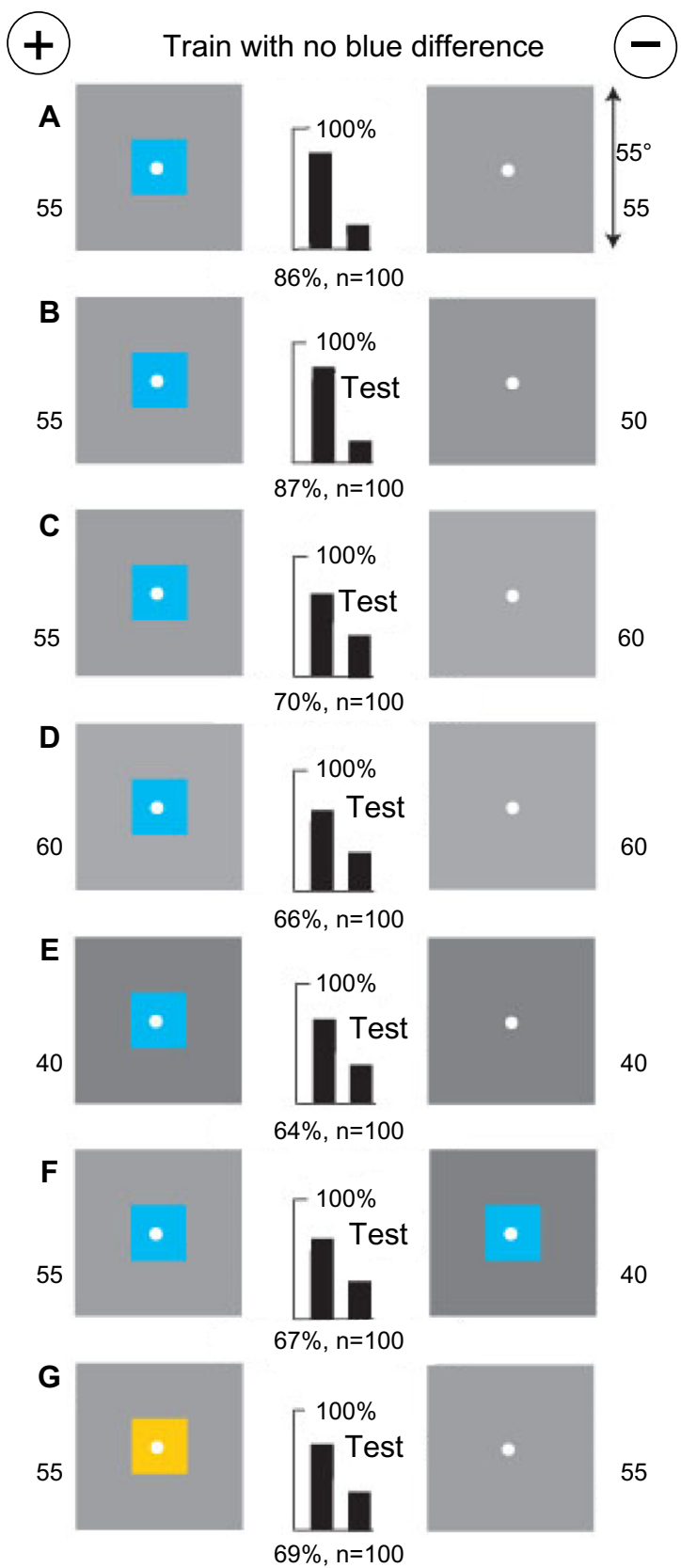

Figure 16 A blue square on a gray background (55\% white), equiluminant to the blue receptors, versus a plain gray target ( $55 \%$ white).

Notes: (A) Training patterns. Green contrast at the edge of the blue was the only detectable difference. (B-E) Various gray levels had no effect because there was always green contrast on the rewarded target. (F) With a blue square on both targets, the expected measure of green contrast was preferred. (G) A buff square provided green modulation but removed some blue content. Gray levels were indicated by the percentage of white, not black, as indicated by numbers at the sides.

\section{With no green contrast or color difference, bees discriminate between positions of a small panel by location of blue content and blue modulation}

A new group of bees was trained to discriminate a small buff square in the right lower corner of a blue background from a similar panel in the left lower corner (Figure 17A). When both panels were moved up, the bees still succeeded, but with a reduced score (Figure 17B). With a yellow border $2^{\circ}$ wide around each panel (Figure 17C) or with blue panels on buff background (Figure 17D), the scores were also reduced. However, with white panels (Figure 17E), they reversed their preference because the average position of blue in each target was reversed (compare Figure 15D and F). With just the edges alone, outlined in blue contrast, the trained bees could just discriminate the positions although there was no difference in the average position of blue content (Figure 17F and G). Therefore, the bees had learned positions of both blue content and blue modulation, irrespective of contrast reversal.

After training a new group of bees with the small panels at the top (Figure 17H), the tests were repeated. Moving the panels down (Figure 17J) or adding a border of strong green contrast (Figure 17K) reduced the score. With black (Figure 17L) or white (Figure 17M) panels on a blue background, the trained bees were lost, but with just the blue modulation at the edges (Figure $17 \mathrm{~N}$ and $\mathrm{P}$ ), they performed very well. Therefore, a measure and position of blue modulation was a strong cue, but average position of blue content was not learned in this example. In these examples, the trained bees learned nothing via the green receptor pathway, but unexpected green contrast reduced the response to blue modulation (Figure 17C and $\mathrm{K}-\mathrm{M}$ ). They would have to compare both targets to do the task.

\section{Bees discriminate between patterns by a measure and location of blue modulation}

A new group of bees was trained to discriminate a buff square on a blue square background from a blue square on a buff square with no green contrast, each on a white background (Figure 18A). The patterns were devised with equal areas of color and exactly the same positions and measures of green modulation on each target. Even the boundaries between blue and white and buff and white had identical green modulation. There was no polarity within either target, leaving no difference to detect. So, as in previous work, ${ }^{16-19}$ a vertical black bar $2^{\circ}$ wide was drawn in the center of each inner square to generate a distinguishing feature, although at identical locations. The bees now had available a difference in blue modulation adjacent to the black bars. They were slow to recognize it.

When the black bars were omitted (Figure 18B), the trained bees failed, showing that the different contrasts against the white background were not the cue and suggesting that different blue contrasts at the sides of the black bars were essential. A test showed that the black bar on the 


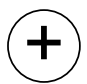

\section{Train with equal blue content} and no green contrast

A

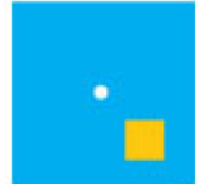

B

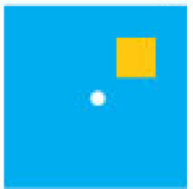

C

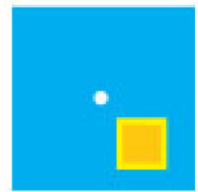

D

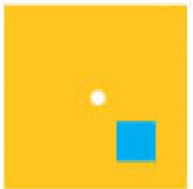

E

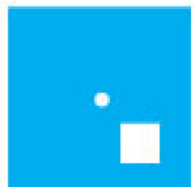

F

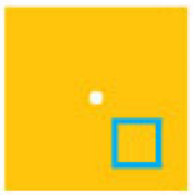

G

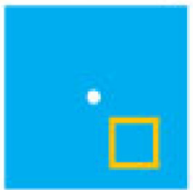

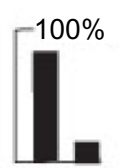

$89 \%, n=100$

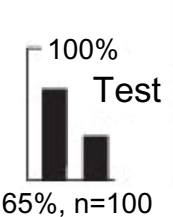

$65 \%, n=100$

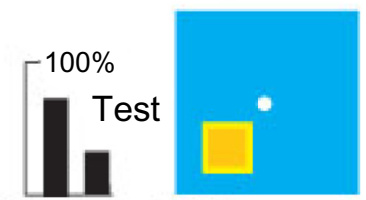

$72 \%, n=100$

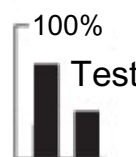

$60 \%, n=100$

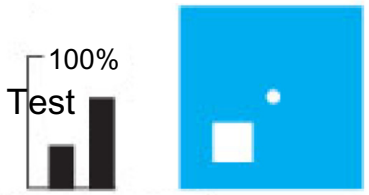

$34 \%, n=100$

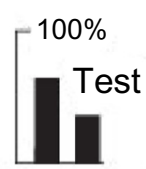

$67 \%, n=100$

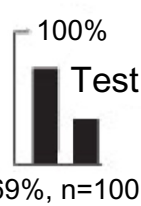

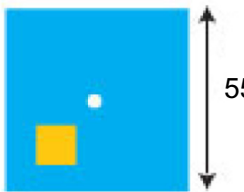
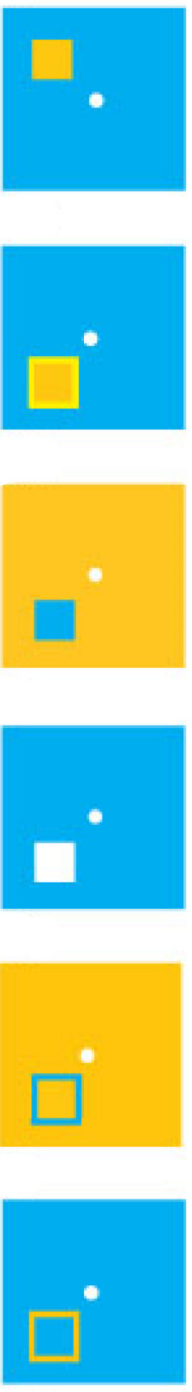

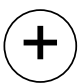

Train with equal blue content and no green contrast

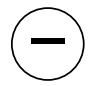

H
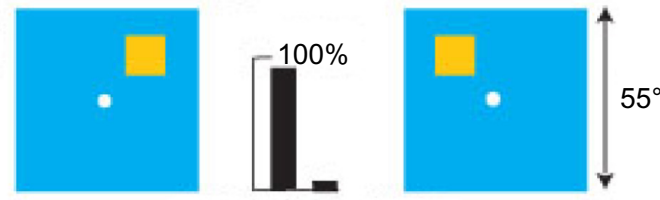

$\mathbf{J}$

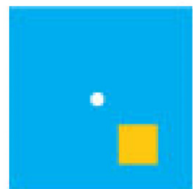

$95 \%, n=100$

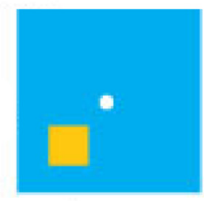

K
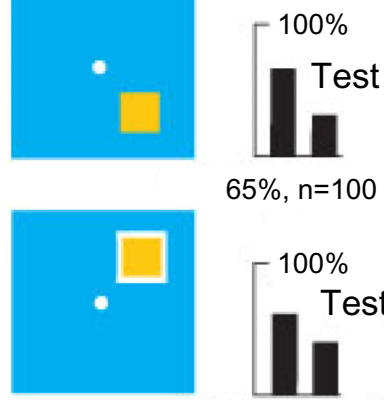

$65 \%, n=100$

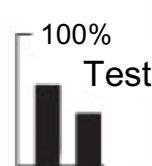

$59 \%, n=100$

$\mathbf{L}$
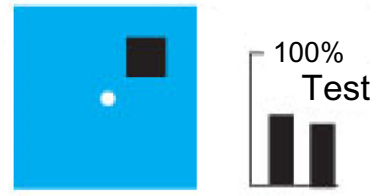

$54 \%, n=100$

M
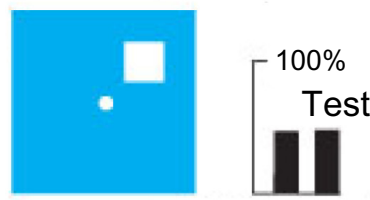

$49 \%, n=100$

$\mathbf{N}$

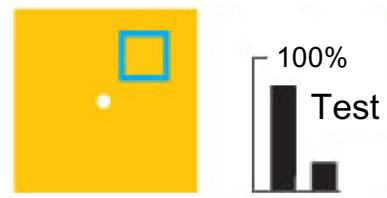

$78 \%, n=100$
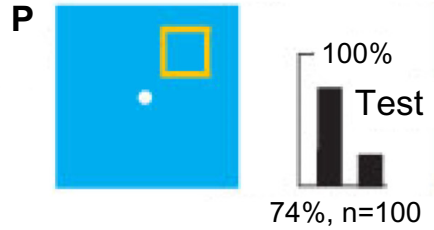
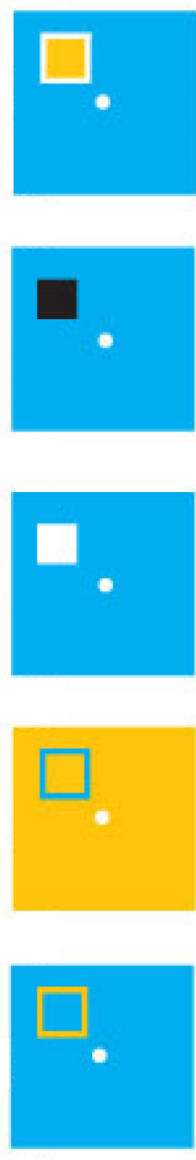

Figure 17 With no green contrast, bees discriminate between positions of a small panel by location of blue content and blue modulation.

Notes: (A) Training patterns. (B) When both panels were moved up, the score was reduced. (C) With a yellow border $2^{\circ}$ wide around each panel or (D) with blue panels on buff background, scores were also reduced. (E) However, with white panels, they reversed their preference. (F and $\mathbf{G})$ With just edges in blue contrast, the trained bees discriminated well, irrespective of contrast reversal. $(\mathbf{H})$ New training targets, with small panels at the top. (J) Moving the panels down or $(\mathbf{K})$ adding a border of green contrast reduced the score. (L) With black (L) or $(\mathbf{M})$ white panels on a blue background, the added green contrast destroyed the memory, but $(\mathbf{N}$ and $\mathbf{P})$ with blue modulation only, they performed very well.

rewarded target was not required (Figure 18C), but the test failed when the bar on the unrewarded target was omitted (not illustrated). They avoided a blue-on-buff square with a black bar in favor of a plain green square (Figure 18D) but failed to recognize buff-on-blue square with bar versus the green square (Figure 18E), showing that they had not learned the rewarded target or any color difference. In a test with two vertical buff bars on blue, with blue contrast but no green contrast, they avoided the width corresponding to the inner edges of the squares (Figure 18F). In a test with gratings with no green contrast, they avoided the greater blue modulation (Figure 18G). 
$\uparrow$

Train with black bars, no difference in average color, and no green contrast

A
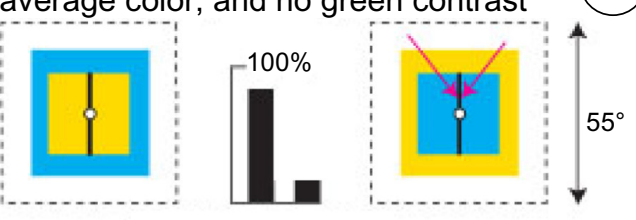

B

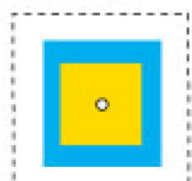

$84 \%, n=200$

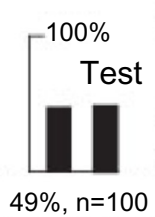

C
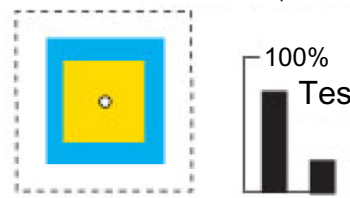

$76 \%, n=100$

D

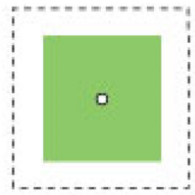

E

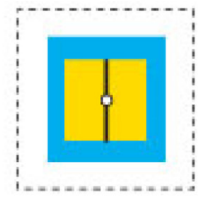

$\mathbf{F}$

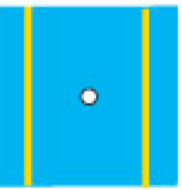

G

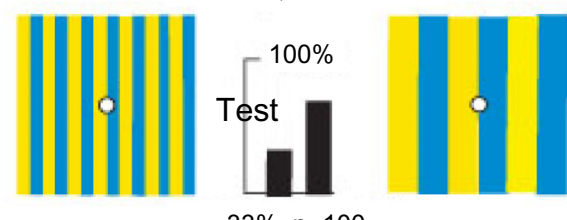

$33 \%, n=100$

Figure 18 Pattern discrimination by measurement and position of blue modulation. Notes: (A) Training patterns of buff and blue, with no green contrast, equal amount of blue, and a central black vertical line on each pattern. (B) Removal of the black bars spoils the discrimination. Nothing on these targets was recognized. (C) The black bar on the unrewarded target was essential. (D) Green can be substituted for equal areas of blue and buff. $(\mathbf{E})$ The rewarded target was not learned. $(\mathbf{F})$ The trained bees avoided bars of blue modulation that were closer together. (G) Testing with blue/buff gratings, they avoided the greater blue modulation. Therefore, they learned to avoid the greater blue modulation at the unrewarded black bar (arrows).

Reexamination of the training patterns in the light of these results shows that the bees avoided blue modulation at the black bar on the blue square (arrows), which was stronger than that on buff. They detected only the blue modulation at the line, ignored the patterns, and learned nothing from the rewarded target. This result also illustrates how bees can be forced to rely on blue modulation when the preferred inputs are denied by colors, equal green modulation, and symmetry of the patterns.

\section{Discrimination between two shapes with no color difference by a measure and position of the strongest green modulation}

Training a new group of bees to discriminate between a yellow diamond (rewarded) and a yellow square of the same size, both on a black background, (Figure 19A) was notably slow, probably because there was no difference in color and blue contrast was low, although green modulation was strong at the vertical edges (Table 1). Tests with each training pattern versus black showed that the trained bees disliked plain black but avoided the unrewarded target even more (Figure 19B and C). However, they distinguished isolated edges very well (Figure 19D). They avoided the greater measure of modulation, irrespective of shape (Figure 19E-G), and the expected modulation position was more relevant than corners, shapes, or yellow areas (Figure 19H and J-L). They detect and avoid the vertical edges of green modulation. Addition of an outer surround of strong blue (in the white) reduced the score (Figure 19M). When tested with gratings equiluminant to blue, with a difference in period, the trained bees avoided greater green modulation (Figure 19N) but detected no difference between gratings equiluminant to green receptors (Figure 19P).

These two training shapes differed in only one set of features that the bees could detect: green modulation. They avoided the greater green modulation at the vertical edges of the unrewarded pattern, irrespective of shape (Figure 19D-G, $\mathrm{J}, \mathrm{M}$ and $\mathrm{N}$ ). To the bees, a measure of contrast was irrelevant because it was the same on each pair of targets, and they did not insist on an absolute measure of green modulation, but avoided the target with the most. Although two shapes were distinguished, some tests showed that neither target was recognized (Figure 19F and K). The bees' visual system was not interested in squares, diamonds, or abstract shapes, but looked for the preferred feature difference.

\section{Discussion}

\section{No sign of trichromatic color vision}

The experimental results above could never be considered as an addition to the current weight of tradition that bees have trichromatic color vision. In every pattern that was examined here and in four preceding papers, ${ }^{16-18,20}$ discrimination was 
Train with green contrast

but little blue content

A
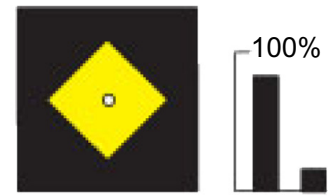

$84.0 \%, \mathrm{n}=200$

B
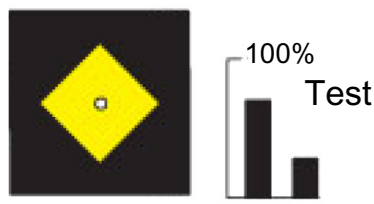

$72 \%, n=100$

C

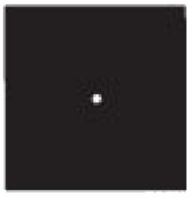

D
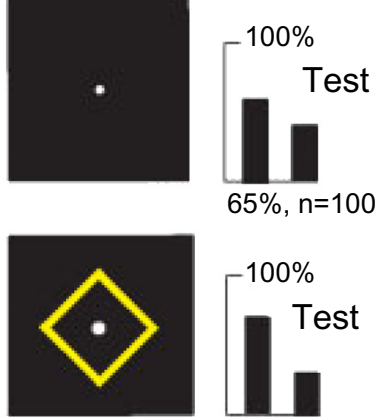

$65 \%, n=100$
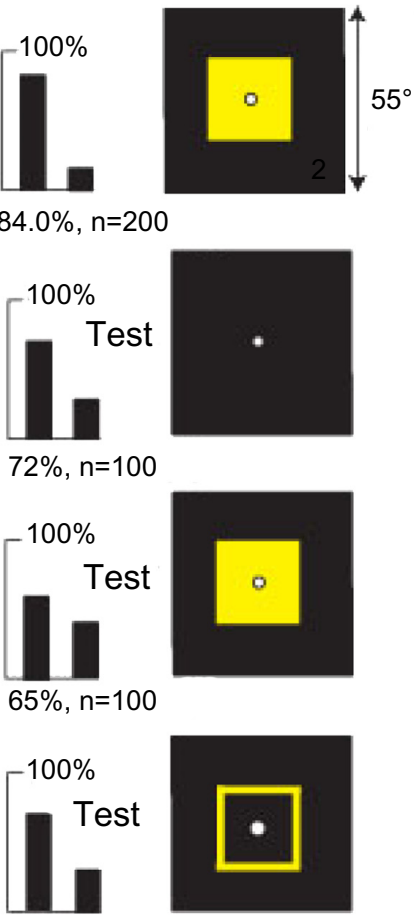

E
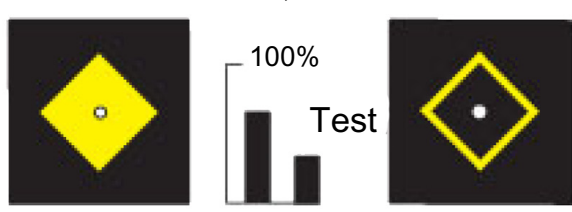

$67 \%, n=100$

$\mathbf{F}$
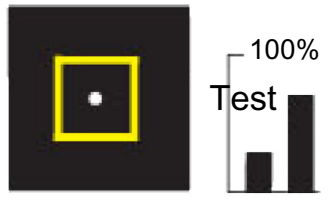

$27 \%, n=100$

G
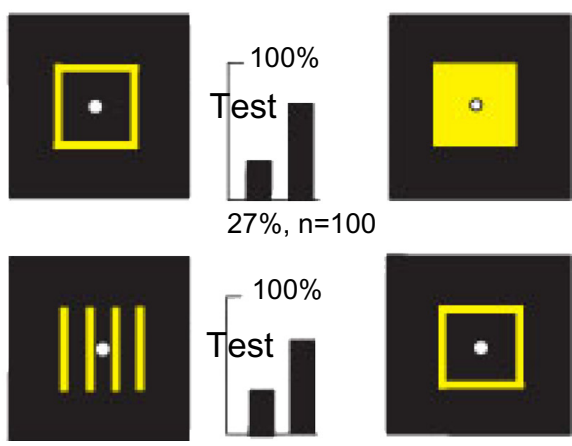

$34 \%, n=100$

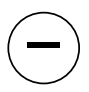

Continued

H
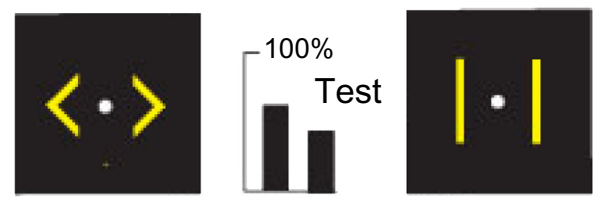

$61.5 \%, n=200$

J
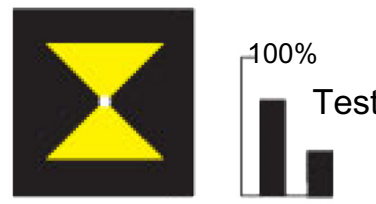

$69 \%, n=100$

K
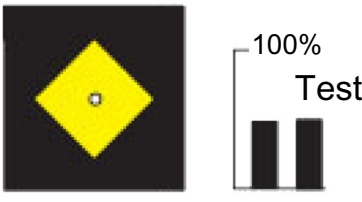

$49 \%, n=100$

L
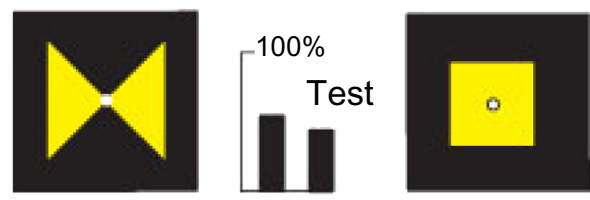

M

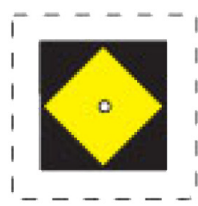

$55 \%, \mathrm{n}=100$
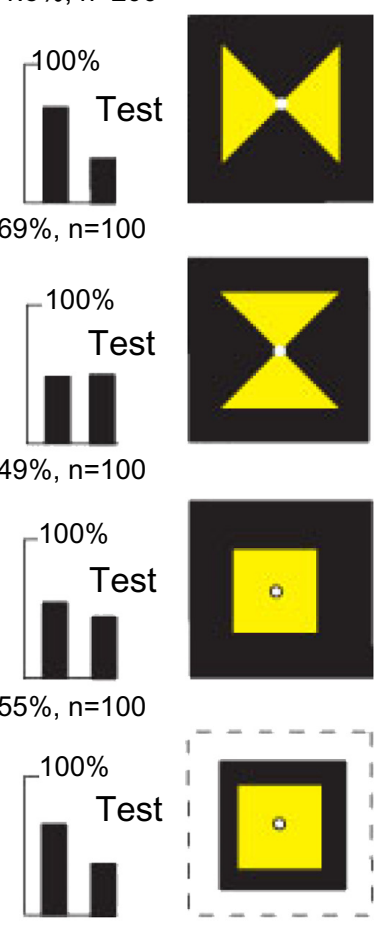

$65 \%, n=100$

$\mathbf{N}$
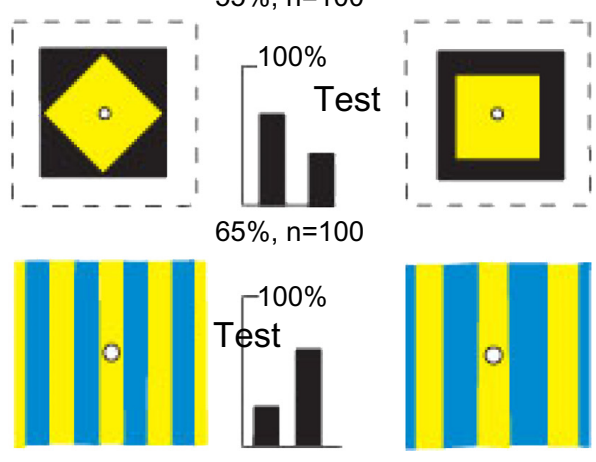

$31.0 \%, n=200$

$\mathbf{P}$
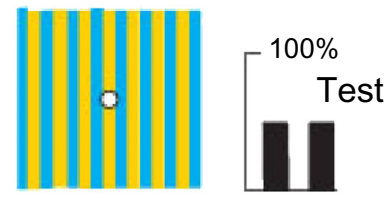

$49.5 \%, n=200$
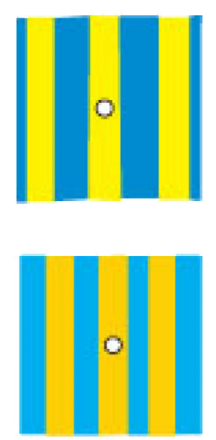

Figure 19 Discrimination of shape by a measure and position of green modulation on the unrewarded target.

Notes: (A) Training patterns with little blue content but strong green contrast. (B and $\mathbf{C}$ ) Tests with each training pattern versus black. Trained bees disliked black but avoided the unrewarded pattern even more. (D) They distinguished edges only. (E- $\mathbf{G})$ They avoided the greater measure of modulation. (H and J-L) The expected green modulation and its position were more relevant than corners, shapes, or yellow areas. (M) Addition of an outer surround of strong blue (in the white) reduced the score. $(\mathbf{N})$ With gratings equiluminant to blue, the trained bees avoided greater green contrast but $(\mathbf{P})$ detected no difference between gratings equiluminant to green receptors.

achieved by locating and measuring blue content and green modulation, with a lower preference for blue modulation. Responses to these three features could be detected, summed over local regions of the eye, and at the same time, the exact locations of vertical edges and angles between them were learned and later recognized. Unique detail could be detected, measured, and learned, while features that conveyed nothing were ignored. Each cue was usually a coincidence between responses to two of the strongest signals. A few cues were remembered, while the numerous responses to individual features were lost in summation, in some cases over the whole of an eye. There are no bee colors, only shades of blue, 
and no achromatic vision of gray or white. Patterns, shapes, symmetry, and colors were of no interest, except as a source of the three types of preferred inputs.

\section{Modulation, not contrast}

Psychophysics of human vision is concerned with contrast, shading, and boundaries between areas of color. By definition, contrast is outside the eye and modulation of the detectors is the response inside the eye as a contrasting edge is scanned. Contrast has polarity, which bees do not detect, ${ }^{17}$ and is independent of image structure. In early artificial seeing systems, ${ }^{21}$ edges were detected with symmetrical detectors that measured the position and depth of contrast at an edge but were also blind to the direction of contrast and were color-blind. On the other hand, in the bee, modulation is a measure of rate of change that depends also on the amount of edge that is scanned. Therefore, image structure in the bee is inseparable from image color, and there is no bee spectrum, only black and shades of blue.

In 1934, Hertz ${ }^{6}$ found that two shapes or simple patterns are more readily distinguished the more they differ in the total length of edge or pattern disruption, called figural intensity, which is now interpreted as modulation of the receptor responses and recognized as the most preferred signal in insect vision. Hertz ${ }^{22}$ distinguished between color training and figural training, corresponding to newly defined inputs of blue content and green modulation. Color training was blocked by UV, but figural training was unaffected. ${ }^{22}$
Hertz also recognized that any stimulus displaced a black or white background. ${ }^{22,29}$

In the honeybee, the signal is carried mainly by measured modulation of the detectors of edges and boundaries in the green receptor pathways. Blue receptor modulation is also located with high resolution and measured in its own separate channels, but its effect is inhibited by the green channels (Figures 7B, F and 17C). The minimum necessary inputs deduced from behavior are surprisingly few (Figure 20) because the analysis has been made in terms of minimum independent variables, but in real nervous systems, no doubt, many possible combinations of these extremes exist possibly with their own distinct signals.

Bees sum these inputs in local areas and forget responses of individual feature detectors. They also detect positions of hubs of radial spokes or tangential curved contours. They learn an averaged signal in a local region, and individual orientations of features are lost. ${ }^{23}$ Committing only totals and averages to memory reduces the information load. After all, they recognize a few landmarks and the angles between them, not every leaf and twig. They recognize signals at the locations where they were in the training. ${ }^{23}$

\section{Coincidences of responses to colored features}

In recent works, we found that bees locate the average position of blue relative to a landmark of green contrast. ${ }^{17}$ They also measured area and intensity of tonic blue content

Three independent variables are sufficient for vision of color
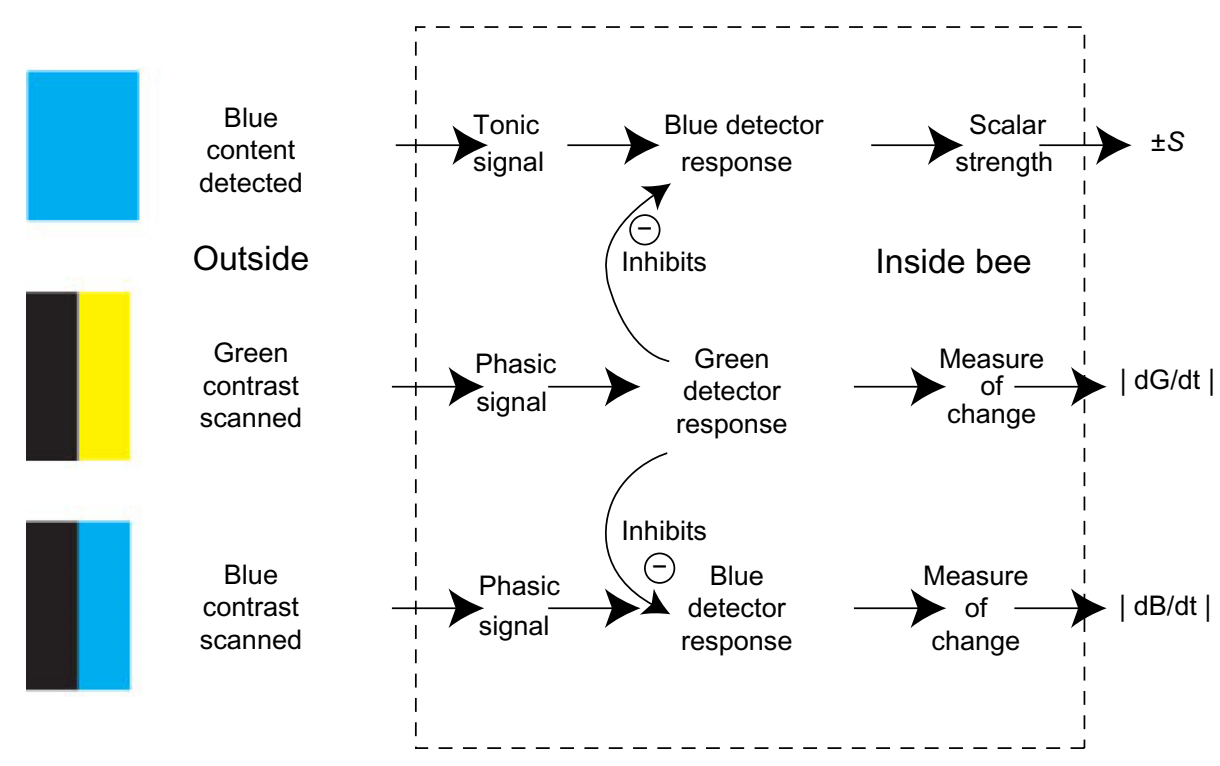

Figure 20 Three neural channels were sufficient and necessary to account for all the experiments.

Notes: Three interacting channels fooled researchers for a century because two detected only edges and experimental results were anti-intuitive. The blue tonic channel reports a blue signal less or greater than background, while the other two channels detect edges irrespective of polarity. Inhibition of a blue input by UV was observed long ago. ${ }^{29}$ Abbreviation: UV, ultraviolet. 
(Figures 8, 15 and 16), but the exact metrical detail was not defined. They measured angular width between two vertical edges as a natural consequence of their separation on the eye (Figures 2, 3, 7 and 10). They also measured the height of the center of an area, ${ }^{10}$ probably as a measure of range. ${ }^{24}$ Bees also detected left-right asymmetry in the horizontal direction by the spatial relation between an area that displayed blue and a region of modulation. ${ }^{17}$ Whether they also detected left-right asymmetry in other ways is an interesting question, now partially answered (Figures 13 and 14).

In the new work, bees compared the widths of two single bars or the outside widths of two groups of bars by green modulation alone (Figures 2 and 3) or by blue modulation alone (Figure 7). Measurement of total modulation, as distinct from local contrast, was demonstrated for the green and blue channels (Figures 4C and 5C). Inhibition of blue modulation by green modulation was also demonstrated (Figures 5E, 7B, $\mathrm{F}, 17 \mathrm{C}, \mathrm{K}$ and $\mathrm{L}$ ). When trained on one pattern versus a plain target (Figures 6, 8, 9, 15 and 16), bees looked first for a difference in blue content and used the same green and blue modulation as with one pattern versus another (Figures 4, 5, 10-12 and 17-19). As previously well documented with black and white patterns, ${ }^{10}$ discrimination of patterns with identical color content was achieved by one or two measures of green modulation. Gray levels provided just another measure of blue content and green modulation at boundaries (Figures 11C, 12, 15 and 16). Local blue content was measured as greater or less than background (Figures 15 and 16). When blue content and green modulation differences were excluded, the bees detected and measured blue modulation (Figure 18). The cues would be little affected by changes in background illumination. Modulation signals therefore play a very large part in bee vision, and with the help of appropriate training patterns, it may be possible to discover new coincidences of cues to extend our model of the bees' visual world.

\section{System of preferences}

A system of preferences dominates vision of the honeybee and all natural and artificial visual systems, because most of the thickly scattered features in the panorama must be ignored. Summation and sparse sampling are ways to avoid the combinatorial explosion when the number of coincidences becomes unmanageable. Bees detect first the strongest green modulation and blue content for the task in hand. Average orientation of edges in a local area is the least preferred input. Therefore, the same few feature responses turn up in every experiment. We can never discover whether bees detect other features that have lower priority.

\section{Recognition of location of cues}

There was a doubt concerning the learning and memory of locations on the targets because the exact direction of the bee gaze was not recorded. Location of a thin vertical bar was remembered with a resolution of approximately $4^{\circ}$ (Figures 2E, 10, 14 and 18), but it was impossible to decide if the memory had a fixed position on the eye or if location was remembered relative to vertical edges elsewhere in the apparatus. In all experiments with vertical edges on stationary targets, bees use a memory of edge location wherever they can (Figures 2, 3, 6, 7, 13, 14 and 17). When bees were tested on targets with an unexpected feature added or something missing, they consistently failed to reach the score in the training, showing that some bees noticed the change. Probably, edges were located relative to large landmarks, especially edges in the apparatus that also briefly stabilized the eye, so that memory persisted where it was learned on the eye and appeared to be retinotopic.

For many years, to avoid this spatially localized memory, work with black and white targets involved shuffling or spatially randomizing on the targets all features that the bees might learn. ${ }^{10}$ The bees became familiar with this requirement and looked for cues irrespective of location. The cues in shuffled targets turned out to be the same as in fixed targets.

\section{Consequences of the training procedure}

In most of the 20th century research, bees approached and inspected targets closely, so there was no control of viewing position or angular size. It was easy to measure performance in training but impossible to discover what bees actually detected because they refused to respond to unexpected test patterns. Nevertheless, the results are usually treated as comparable with each other despite the variety of procedures. From 1986, with patterns alternating between the two sides of the Y-choice maze (Figure 1), bees responded well in tests. They surveyed the test targets and searched for cues at a defined range.

The bees were always presented with two targets, although one may be plain white, black, or colored. In one strategy, called differential conditioning, the bees were presented with a pattern on each target and learned by trial and error, so they first learned most from their errors on the unrewarded side, unless both targets were essential. Bees learned two or three preferred features that they detected, located, and measured. They learned first the position and measure of the strongest input. When a cue was present in the same position on both targets, they learned to ignore it. When they returned for more 
reward, they recognized the little they had learned, not the entire pattern, or the total difference between two patterns.

In the other strategy, absolute conditioning, the bees chose a rewarded pattern when the alternative was blank or a continuation of background. With this method, bees located green contrast, measured blue content, and learned first to avoid the unrewarded target exactly as they did with two targets (Figures 6, 8, 9, 15 and 16).

With black and white patterns ${ }^{10}$ or colors, ${ }^{16-18}$ bees detected the same features with the same preferences, irrespective of training strategy. The bees' strategy is not conditioning in a classical sense used by Pavlov or Jung, in which the reward was supposed to initiate the unlikely recall of an unrewarded transitory image and fix it into memory. Bee vision requires active scanning that repeatedly generates the modulation of the receptors, called operant conditioning by Skinner, together with trial and error learning as described by Jennings, Thorndike, and others long ago. ${ }^{25}$

\section{Consequences of the testing procedure}

When trained bees return to the choice chamber, many detect nothing to avoid and continue on their flight path to the reward. After all, a score of $80 \%$ implies that $40 \%$ of the choices were made at random. Most arriving bees stop approximately $2 \mathrm{~cm}$ from the baffle and either hover or scan. Some hover where they see both targets. The bees search first one target and then the other, acting as if making a choice; hence, the testing procedure seems likely to reveal a memory if there is one.

Each test shows whether the bees are successful or not, which is not of much use unless many varied tests are done. This method will not discover what the bees have detected but failed to learn. Each test is an independent forced test, so the score should be $50 \%$ or $100 \%$ correct if the bees all behave in the same way. Clearly, they do not.

The most effective tests are those in which the bees fail, because it is then certain that the bees recognized nothing in the display or opposing features canceled.

In these experiments, learning and memory apply to the pair of training targets at that place. If anything is changed, the bees start to retrain themselves by trial and error. Therefore, it is essential to retrain after every test and convenient to have a succession of many different tests. The richness of bee vision is more theoretical than real, because bees learn only the task in hand and the sampling is very sparse and sufficient only to make that particular choice. The limitations of the Y-choice maze were that one task was isolated and analyzed as if variables were separate - although in reality, the location, image structure, blueness, background, and green modulation were intimately linked - and a sequence of bee choices was not analyzed along a route.

\section{Do bees recognize symmetry or asymmetry?}

In the previous work, it was shown that the relative positions of a blue area and a green modulation acted as a directional signpost with polarity. ${ }^{17}$ In the new work, with only green contrast available, bees learned to locate the position of the strongest concentration of green modulation on the unrewarded pattern, irrespective of which pattern was symmetrical (Figure 14). There was no evidence that they distinguished abstract symmetry about a vertical axis versus a similar asymmetrical one. In other demonstrations of discrimination of symmetry, there was no analysis of modulation; symmetrical patterns were rewarded and the unsymmetrical ones were unrewarded, and it was concluded that the bees recognized symmetry, but probably they learned to avoid familiar cues, irrespective of abstract symmetry.

\section{Use of gray levels by von Frisch}

During the 19th century, excellent observers studied bees' choices of flower colors and concluded that they learned landmarks not colors. ${ }^{26}$ In 1912, Carl von Hess, professor of Ophthalmology at Münich, concluded from the available data that bees did not have color vision like humans. ${ }^{27}$ A young assistant in the Zoology Department, von Frisch, ${ }^{1}$ immediately set to work to prove that bees pass the standard test for human color vision.

Von Frisch laid out a series of 32 (later 16) gray papers from white to black in random order on a table. He failed to train bees to come to mid-gray versus the rest laid out in close rectangle, but black or white was distinguished. He had no explanation. Next, he trained them to come to a colored paper versus the gray series. ${ }^{1}$ Except for greenish-blue or green, they learned to ignore the gray papers. Von Frisch accepted this as proof of human-like color vision.

His bees could not pick out mid-gray from his gray series, from which we conclude that they had learned the average blue level in the whole gray series, which was similar to the blue level in mid-gray. To his bees, each color displayed a different measure of blue and also modulation of green and blue receptors at edges. For example, yellow paper would appear black to the blue receptors but would display strong green modulation at its edges with gray. Von Frisch thought that they saw individual gray levels and learned yellow, but actually they learned the lack of blue. Von Frisch never understood his own results. 
Unfortunately, the test for defects in human color vision was not a suitable tool to investigate an unknown system. Successful performance alone said nothing about mechanisms, and any arbitrary theory that fitted the results could not be disproved. A small anomaly, like the failure to distinguish green, was ignored.

Von Hess had a different training arrangement, ${ }^{28}$ with eight blue squares and eight yellow squares arranged in a checkerboard. He trained on several blue squares, or alternately on yellow squares, and then tested by introducing other colors, black, or white. Von Hess was unaware that bees trained on yellow squares learned green modulation and avoided blue. Their responses in tests with other colors appeared very strange, because everyone believed they had learned to go to yellow. Von Hess contradicted von Frisch but never understood his own results either.

\section{What is blue content?}

In general, the word blue refers to the stimulus that excites the blue receptor channel, which includes quite a wide spread in the wavelength of the light, depending on intensity. Almost every experiment showed that bees located and measured blue on each target, but for two reasons the details of the measurement were not clear. Most experiments demonstrated a summation of tonic blue over large fields (Figures 15 and 18), but there was also discrimination of local blue position (Figure 17B and E) and the apparatus limited the target size to a maximum $55^{\circ}$. Additionally, the relationship with the brightness of blue and the part played by coincident UV light have not been explored recently, although Hertz ${ }^{29}$ reported a large inhibitory effect of UV on responses to white (see Figure 21).

\section{Attention and selection}

Even the most primitive animals react to one stimulus when several are present which would, if acting alone, also produce a response. ${ }^{25}$ An obvious inference is that the initiation of one response inhibits the alternatives. In some cases, this clearly happens in the bee (compare Figure 21). Responses to blue modulation, for example, are inhibited by the presence of green modulation (Figures 7B, F, 17C and K) and responses to blue are inhibited by $\mathrm{UV}^{22,29,30}$ It is more difficult to determine whether responses to summed inputs from a large field are active at the same time as local inputs from the same field

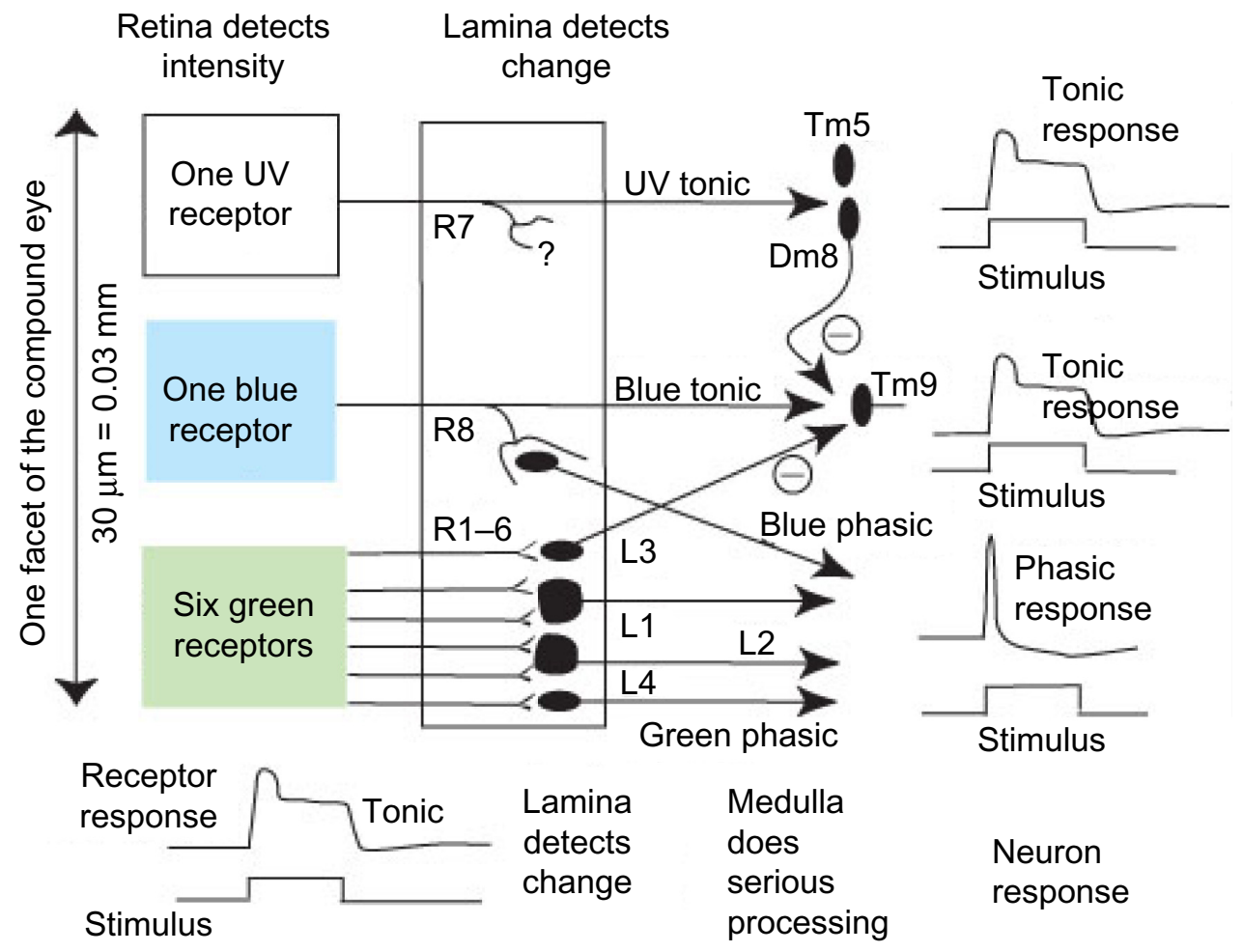

Figure 21 A neural substrate to relate detection of color to behavior.

Notes: Six green-sensitive receptors, and one each of blue and UV, detect a measurement of tonic (maintained level) intensity (left and lower left). At the lamina (center), the next neuron layer in the optic lobe, six green-sensitive receptors all end on second-order neurons that adapt rapidly, therefore respond only to changes in the input. The blue receptor sends a phasic as well as a tonic signal. Inhibition of a blue input by UV was observed long ago. ${ }^{30}$ Labels on neurons are those commonly employed for identified neurons in the fly Drosophila.

Abbreviation: UV, ultraviolet. 
of view; for example, blue content was summed over a large angle while there was local retinotopic blue modulation in the same field (Figure 17). All colors, apart from black and saturated yellow, display blue modulation as well as blue content, so shape and color continually interact with each other.

\section{Neural substrate}

With the new information, ${ }^{16-18}$ a formal model of the identified inputs can be based upon the known physiology ${ }^{2,4}$ of the insect optic lobe and anatomy of Drosophila, as a convenient way of summarizing many conclusions from two decades of testing trained bees. Light is detected by three receptor types. At the left (Figure 21), in each ommatidium are six green receptors, one blue receptor, and one UV receptor. In the training experiments, UV was excluded to reduce the number of variables in the analysis. The response of the receptor cells is a steep electrical response at "on", followed by a high plateau (bottom left), so the input preserves a maintained level of intensity. At the next neuron layer in the optic lobe, the lamina (Figure 21, center), the six green-sensitive R1-6 receptors all end on second-order neurons that adapt rapidly and respond to changes in the input. ${ }^{4}$ Effectively, they transmit a temporal derivative of the input signal and are sensitive to edges, lines, and boundaries. ${ }^{11}$ The blue receptor output has a similar transformation of the signal but in addition passes through the lamina to the medulla, so there are two blue inputs, tonic and phasic, from each ommatidium. The UV receptor axon also goes directly to the medulla. Modulation therefore begins at the lamina level in both green and blue channels, there is no tonic green input, and UV was excluded. Coincidences and summations of these inputs begin in the medulla of the optic lobe. In the deep optic lobe of the bee, the properties of neurons with wide fields antagonistic inputs from two or three receptor channels agree very well with the present results. These neurons arise by the addition and/or subtraction of the three peripheral inputs of Figure 20 in different proportions in local regions or over the whole eye..$^{31}$ Each is specific for one combination or coincidence of inputs, and this whole group of neurons divides the input into specific items ready to be fed into memory. They account very well for the way bees distinguish colored patterns.

It is amazing and satisfying that hundreds of decisions by bees, in a great variety of visual tasks, can be explained by these few superficial pathways (Figure 21). The diversity of patterns was possible because there was also a quantitative measure of the stimulus in each channel and of angles between coincident responses. A location and mechanism for these measurements and coincidences is not yet known.

\section{Is this an inevitable visual system for an insect}

After the demonstration that a visual system with only monochromatic blue content plus green and blue phasic channels explains successes and failures of recognition (Figures 20 and 21), it seems unlikely that any insect will have trichromatic color vision. Many insects and crustaceans also have six green receptors in each ommatidium and two that are sensitive to blue or UV.

Being a herbivore, the worker bee needs to identify places by landmarks and sense whether a flower is more or less blue than a green background, but some insects have a more demanding lifestyle. Dragonflies and some butterflies have a greater variety of receptors, but there is no reason to infer that they have polychromatic color vision. More likely, they evolved extra receptor types to improve detection of a food plant or a mate. A similar system as the bee can be expected in other insects and perhaps all arthropods.

\section{What does the honeybee see?}

A new feature of the bee visual system is the coexistence of a tonic blue input that depends on area and two modulated inputs that depend upon the length and locations of edges as well as contrast against background. Therefore, the apparent structure of the image is inseparable from the distribution of color, black, and white, while responses to color differences depend on spatial structure and the background.

Bee vision of blue is more like our skin senses that can locate a sharp edge and simultaneously radiant heat. All our sensory inputs except vision make use of cross-modal coincidences of their responses. In a similar way, bees recognize the identity of a place by feeling a coincidence of responses of feature detectors in different dimensions that form no image.

Measurements of resolution of the feature detectors for each receptor channel are already well-known. ${ }^{13,14}$ Although flower color, shape and length of edge, are inseparably linked in visual processing, the resolution or discrimination of small differences is improved by restricting the system to the strongest input. For the plants, this implies that constancy of flower shape and pattern is just as important as constancy in flower color.

We cannot assume that just because bees relate to the natural panorama in a sensible way, as if they see something, they must see shapes and colors of flowers. We observe a blind man washing, dressing, shopping, or preparing a meal and acting as if he sees, but we cannot infer that he sees like the rest of us. Human vision is not the way bees see a panorama, but my final comment is that human vision functions 
like a bee, with tonic receptor responses and coincidences of phasic modulation derivatives at edges, because it is a neuron machine.

\section{Acknowledgment}

Thanks to Richard Johnston for the loan of a hive of bees.

\section{Disclosure}

The author reports no conflicts of interest in this work.

\section{References}

1. von Frisch K. Der Farbensinn und Formensinn der Bienen. [The color and shape sense of bees]. Zool Jahrb Physiol. 1914;35(1):1-188. German.

2. Autrum H, von Swehl V. Die Spektralempfindlichkeit einzelner Sehzellen des Bienenauges. [Spectral sensitivity of some photoreceptors of the eye of the bee]. Z Vgl Physiol. 1962;48:357-384. German.

3. Baumann F. Electrophysiological properties of the honey bee retina. In: Horridge GA, editor. The Compound Eye and Vision of Insects. Oxford: Clarendon Press; 1975:53-74.

4. Laughlin SB, Hardie RC. Common strategies for light adaptation in the peripheral visual systems of fly and dragonfly. J Comp Physiol A. 1978;128(5):319-340.

5. Srinivasan MV, Laughlin SB, Dubs A. Predictive coding: a fresh view of inhibition in the retina. Proc R Soc Lond B Biol Sci. 1982;216(1205): 427-459.

6. Hertz M. Über figurale Intensität und Qualitäten in der Optische Wahnehmung der Biene. [Pattern density and qualities in the visual perception of the bee]. Biol Zentralbl. 1934;53:10-40. German.

7. Horridge GA. Pattern discrimination by the honeybee, disruption as a cue. J Comp Physiol A. 1997;18:267-277.

8. Wakakuwa M, Kurasawa M, Giurfa M, Arikawa K. Spectral heterogeneity of honeybee ommatidia. Naturwissenschaften. 2006;92:464-467.

9. Kaiser W. The relation between visual movement detection and colour vision in insects. In: Horridge GA, editor. The Compound Eye and Vision of Insects. Oxford: Clarendon Press; 1975:53-74.

10. Horridge GA. What Does the Honeybee See? And How Do We Know? Canberra: ANU Press; 2009. Available from: http://epress.anu.edu.au/ honeybee_citation.html. Accessed April 17, 2015.

11. Srinivasan MV, Lehrer M. Spatial acuity of honeybee vision and its spectral properties. J Comp Physiol A. 1988;181(3):267-277.
12. Giger A, Srinivasan MV. Pattern recognition in honeybees: chromatic properties of orientation analysis. J Comp Physiol A. 1996;178(5): 763-769.

13. Horridge GA. Visual resolution of gratings by the compound eye of the bee Apis mellifera. J Exp Biol. 2003;206(13):2105-2110.

14. Horridge GA. Visual resolution of the orientation cue by the honeybee (Apis mellifera). J Insect Physiol. 2003;49(8):1145-1152.

15. Friedlaender M. Zur Bedeutung des Fluglochs im optischen Feld der Biene bei senkrechter Dressuranordnung. [The significance of the reward hole in the visual field of the bee when training on a vertical target]. Z Vgl Physiol. 1931;15(2):193-260. German.

16. Horridge A. How bees distinguish colors. Eye Brain. 2015;7:17-34.

17. Horridge A. How bees discriminate a pattern of two colors from its mirror image. PLoS One. 2015;10(1):1-23.

18. Horridge A. How bees distinguish black from white. Eye Brain. 2014; 6(1):9-17.

19. Horridge GA. Pattern vision of the honeybee (Apis mellifera): the effect of pattern on the discrimination of location. J Comp Physiol A. 1999;185(2):105-113.

20. Horridge A. Pattern vision of the honeybee (Apis mellifera): blue and green receptors in the discrimination of translocation. Neurobiol Learn Mem. 2000;74(1):1-16.

21. Marr D. Vision. San Francisco: Freeman; 1982.

22. Hertz M. Beitrage zum Farbensinn und Formensinn der Biene. [A contribution on the color and shape sense of the bee]. Z Vgl Physiol. 1937;24(3):413-421. German.

23. Horridge GA. Discrimination of single bars by the honeybee (Apis mellifera). Vision Res. 2003;43(7):1257-1271.

24. Collett TS. Landmark learning and guidance in insects. Phil Trans $R$ Soc Lond B. 1992;337:295-303.

25. Jennings HS. Behavior of the Lower Organisms. New York: Columbia University Press; 1923.

26. Forel A. The Senses of Insects. London: Methuen; 1908.

27. von Hess C. Vergleichende Physiologie des Gesichtsinnes. [Comparative Physiology of Vision]. Jena: Gustav Fischer; 1912. German.

28. von Hess C. Beiträge zur Frage nach einen Farbensinne bei Bienen. [Contribution to the question of color vision in bees]. Pflügers Arch Gesamte Physiol Menschen Tiere. 1918;170(7-9):337-366. German.

29. Hertz M. Versuche über das Farbensystem der Bienen. [Research on bee color vision]. Naturwissenschaften. 1937;30:492-493. German.

30. Hertz M. New experiments on color vision in bees. J Exp Biol. 1939; 116(1):1-8.

31. Yang EC, Lin HC, Yung YS. Patterns of information processing in the lobula of the honeybee. J Insect Physiol. 2004;50:913-925.
Eye and Brain

\section{Publish your work in this journal}

Eye and Brain is an international, peer-reviewed, open access journal focusing on clinical and experimental research in the field of neuroophthalmology. All aspects of patient care are addressed within the journal as well as basic research. Papers covering original research, basic science, clinical and epidemiological studies, reviews and evaluations, Submit your manuscript here: http://www.dovepress.com/eye-and-brain-journal

\section{Dovepress}

guidelines, expert opinion and commentary, case reports and extended reports are welcome. The manuscript management system is completely online and includes a very quick and fair peer-review system, which is all easy to use. Visit http://www.dovepress.com/testimonials.php to read real quotes from published authors. 